\title{
GLOBAL BIFURCATION AND EXACT MULTIPLICITY OF POSITIVE SOLUTIONS FOR A POSITONE PROBLEM WITH CUBIC NONLINEARITY AND THEIR APPLICATIONS
}

\author{
KUO-CHIH HUNG AND SHIN-HWA WANG
}

ABSTRACT. We study the global bifurcation and exact multiplicity of positive solutions of

$$
\left\{\begin{array}{l}
u^{\prime \prime}(x)+\lambda f_{\varepsilon}(u)=0,-1<x<1, u(-1)=u(1)=0, \\
f_{\varepsilon}(u)=-\varepsilon u^{3}+\sigma u^{2}+\tau u+\rho,
\end{array}\right.
$$

where $\lambda, \varepsilon>0$ are two bifurcation parameters, and $\sigma, \rho>0, \tau \geq 0$ are constants. By developing some new time-map techniques, we prove the global bifurcation of bifurcation curves for varying $\varepsilon>0$. More precisely, we prove that, for any $\sigma, \rho>0, \tau \geq 0$, there exists $\varepsilon^{*}>0$ such that, on the $\left(\lambda,\|u\|_{\infty}\right)$ plane, the bifurcation curve is S-shaped for $0<\varepsilon<\varepsilon^{*}$ and is monotone increasing for $\varepsilon \geq \varepsilon^{*}$. (We also prove the global bifurcation of bifurcation curves for varying $\lambda>0$.) Thus we are able to determine the exact number of positive solutions by the values of $\varepsilon$ and $\lambda$. We give an application to prove a long-standing conjecture for global bifurcation of positive solutions for the problem

$$
\left\{\begin{array}{l}
u^{\prime \prime}(x)+\lambda\left(-\varepsilon u^{3}+u^{2}+u+1\right)=0, \quad-1<x<1, \\
u(-1)=u(1)=0
\end{array}\right.
$$

which was studied by Crandall and Rabinowitz (Arch. Rational Mech. Anal. 52 (1973), p. 177). In addition, we give an application to prove a conjecture of Smoller and Wasserman (J. Differential Equations 39 (1981), p. 283, lines $2-3)$ on the maximum number of positive solutions of a positone problem.

\section{INTRODUCTION}

In this paper we mainly study the global bifurcation and exact multiplicity of positive solutions of the positone problem with cubic nonlinearity

$$
\left\{\begin{array}{l}
u^{\prime \prime}(x)+\lambda f_{\varepsilon}(u)=0,-1<x<1, u(-1)=u(1)=0, \\
f_{\varepsilon}(u)=-\varepsilon u^{3}+\sigma u^{2}+\tau u+\rho, \lambda, \varepsilon>0,
\end{array}\right.
$$

where $\lambda, \varepsilon$ are two bifurcation parameters, and

$$
\sigma, \rho>0, \quad \tau \geq 0
$$

are constants.

For any $\varepsilon>0$, it is easy to see that there exist a positive number $\beta_{\varepsilon}$, which is the unique positive zero of $f_{\varepsilon}(u)$, and a positive number $\gamma_{\varepsilon}=\sigma /(3 \varepsilon)<\beta_{\varepsilon}$, which

Received by the editors July 9, 2010 and, in revised form, February 19, 2011 and June 23, 2011.

2010 Mathematics Subject Classification. Primary 34B18, 74G35.

Key words and phrases. Global bifurcation, exact multiplicity, positive solution, positone problem, S-shaped bifurcation curve, time map.

This work was partially supported by the National Science Council of the Republic of China under grant No. 98-2115-M-007-008-MY3. 


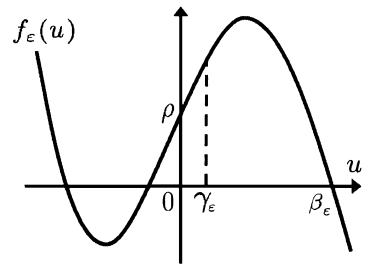

(i)

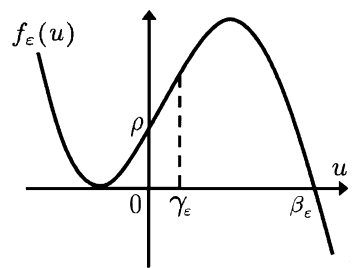

(ii)

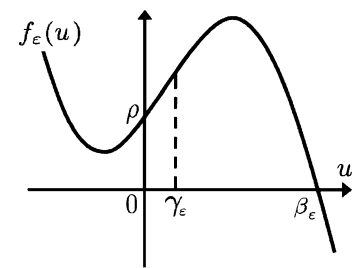

(iii)

Figure 1. Three possible graphs of $f_{\varepsilon}(u)$ satisfying (1.2). (i) $f_{\varepsilon}(u)$ has exactly three real zeros; (ii) $f_{\varepsilon}(u)$ has exactly two real zeros; (iii) $f_{\varepsilon}(u)$ has exactly one real zero.

is the unique (positive) inflection point of $f_{\varepsilon}(u)$, such that the cubic polynomial $f_{\varepsilon}$ satisfies

(i) $f_{\varepsilon}(0)=\rho>0$ (positone), $f_{\varepsilon}^{\prime}(0)=\tau \geq 0, f_{\varepsilon}(u)>0$ on $\left(0, \beta_{\varepsilon}\right)$ and $f_{\varepsilon}\left(\beta_{\varepsilon}\right)=$ 0 ,

(ii) $f_{\varepsilon}(u)$ is strictly convex on $\left(0, \gamma_{\varepsilon}\right)$ and is strictly concave on $\left(\gamma_{\varepsilon}, \infty\right)$. (So $f_{\varepsilon}$ is convex-concave on $\left(0, \beta_{\varepsilon}\right)$.)

Note that it is easy to see that $\beta_{\varepsilon}$ is a continuous, strictly decreasing function of $\varepsilon>0$. In addition, $\lim _{\varepsilon \rightarrow 0^{+}} \beta_{\varepsilon}=\infty$ and $\lim _{\varepsilon \rightarrow \infty} \beta_{\varepsilon}=0$. Three possible graphs of $f_{\varepsilon}(u)$ satisfying (1.2) are depicted in Figure 1.

For any $\varepsilon>0$, on the $\left(\lambda,\|u\|_{\infty}\right)$-plane, we study the shape and structure of bifurcation curves $S_{\varepsilon}$ of positive solutions of (1.1), (1.2), defined by

$$
S_{\varepsilon} \equiv\left\{\left(\lambda,\left\|u_{\lambda}\right\|_{\infty}\right): \lambda>0 \text { and } u_{\lambda} \text { is a positive solution of (1.1), (1.2) }\right\} \text {. }
$$

We say that, on the $\left(\lambda,\|u\|_{\infty}\right)$-plane, the bifurcation curve $S_{\varepsilon}$ is S-shaped if $S_{\varepsilon}$ is a continuous curve and there exist two positive numbers $\lambda_{*}<\lambda^{*}$ such that $S_{\varepsilon}$ has exactly two turning points at some points $\left(\lambda^{*},\left\|u_{\lambda^{*}}\right\|_{\infty}\right)$ and $\left(\lambda_{*},\left\|u_{\lambda_{*}}\right\|_{\infty}\right)$, and

(i) $\lambda_{*}<\lambda^{*}$ and $\left\|u_{\lambda^{*}}\right\|_{\infty}<\left\|u_{\lambda_{*}}\right\|_{\infty}$,

(ii) at $\left(\lambda^{*},\left\|u_{\lambda^{*}}\right\|_{\infty}\right)$ the bifurcation curve $S_{\varepsilon}$ turns to the left,

(iii) at $\left(\lambda_{*},\left\|u_{\lambda_{*}}\right\|_{\infty}\right)$ the bifurcation curve $S_{\varepsilon}$ turns to the right.

See Figure 2(i) for example.

In Theorem 2.1 stated below for (1.1), (1.2) with varying $\varepsilon>0$, we prove the global bifurcation of bifurcation curves $S_{\varepsilon}$ and determine the exact multiplicity of positive solutions by the values of $\varepsilon$ and $\lambda$; see Figure 2. In addition, we give lower and upper bounds of the critical bifurcation value $\tilde{\varepsilon}$.

When

$$
\sigma=\tau=\rho=1
$$

problem (1.1), (1.2) reduces to

$$
\left\{\begin{array}{l}
u^{\prime \prime}(x)+\lambda\left(-\varepsilon u^{3}+u^{2}+u+1\right)=0, \quad-1<x<1 \\
u(-1)=u(1)=0, \quad \lambda, \varepsilon>0 .
\end{array}\right.
$$

Crandall and Rabinowitz [3, p. 177] first considered shapes of bifurcation curves of positive solutions for the $n$-dimensional problem of (1.3),

$$
\left\{\begin{array}{l}
\Delta u(x)+\lambda\left(-\varepsilon u^{3}+u^{2}+u+1\right)=0 \text { in } \Omega, \\
u=0 \text { on } \partial \Omega, \lambda, \varepsilon>0,
\end{array}\right.
$$




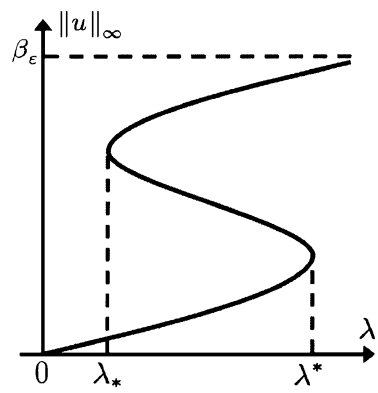

(i) $0<\varepsilon<\tilde{\varepsilon}$

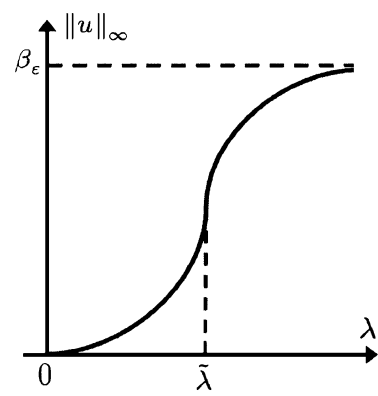

(ii) $\varepsilon=\tilde{\varepsilon}$

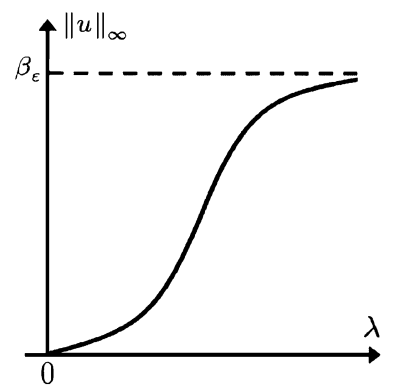

(iii) $\varepsilon>\tilde{\varepsilon}$

Figure 2. Global bifurcation of bifurcation curves $S_{\varepsilon}$ of (1.1), (1.2) with varying $\varepsilon>0$.

where $\Omega$ is a general bounded domain in $\mathbb{R}^{n}(n \geq 1)$ with smooth boundary $\partial \Omega$. They proved that, by using the Implicit Function Theorem and perturbation arguments, when $\varepsilon>0$ is sufficiently small, the bifurcation curve of positive solutions of (1.4) is S-like shaped on the $\left(\lambda,\|u\|_{\infty}\right)$-plane; that is, problem (1.4) has at least three positive solutions for a range of positive $\lambda$. Shi [18, Theorem 4.1] proved that the bifurcation curve of positive solutions of (1.4) is S-shaped when $\varepsilon>0$ is small and $\Omega$ is a ball $B^{n}$ in $\mathbb{R}^{n}$ with $1 \leq n \leq 6$. For (1.3), Brown et al. [2, p. 482] showed that the bifurcation curve $S_{\varepsilon}$ is monotone increasing for $\varepsilon \geq 1 / \sqrt{27} \approx 0.192$ and is S-like shaped for $0<\varepsilon<4 / 27 \approx 0.148$. Wang [21, Theorem 4] used the quadrature method (time-map method) to prove that the bifurcation curve $S_{\varepsilon}$ is S-shaped for $0<\varepsilon<1 / \sqrt{54} \approx 0.136$.

For (1.3), it was conjectured and strongly supported by numerical evidence that, when $\varepsilon$ increases across some critical value $\tilde{\varepsilon}>0$, there exists a cusp bifurcation from an S-shaped curve to a monotone increasing curve on the $\left(\lambda,\|u\|_{\infty}\right)$-plane. More precisely, the following assertions (i)-(iii) hold:

(i) For $0<\varepsilon<\tilde{\varepsilon}$, the bifurcation curve $S_{\varepsilon}$ of (1.3) is S-shaped. Moreover, there exist two positive numbers $\lambda_{*}<\lambda^{*}$ such that (1.3) has exactly one degenerate positive solution $u_{\lambda_{*}}$ and $u_{\lambda^{*}}$ for $\lambda=\lambda_{*}$ and $\lambda=\lambda^{*}$, respectively (see Figure 2(i)).

(ii) For $\varepsilon=\tilde{\varepsilon}$, the bifurcation curve $S_{\tilde{\varepsilon}}$ of (1.3) is monotone increasing. Moreover, problem (1.3) has exactly one (cusp type) degenerate positive solution $u_{\tilde{\lambda}}$ (see Figure 2(ii)).

(iii) For $\varepsilon>\tilde{\varepsilon}$, the bifurcation curve $S_{\varepsilon}$ of (1.3) is monotone increasing. Moreover, all positive solutions $u_{\lambda}$ of (1.3) are nondegenerate (see Figure 2(iii)).

See [2, 18, 21]. Of particular interest are the values $\varepsilon=\tilde{\varepsilon}$ and $\lambda=\tilde{\lambda}$, at which two nondegenerate turning points coalesce into a single degenerate turning point. This point is often called a double turning point (cf. Tanaka, Murashige and Oishi [20]). Note that the definitions of degenerate and nondegenerate positive solution are given in Section 3 below.

In Corollary 2.2 stated below for (1.3), which follows from Theorem 2.1, we prove this conjecture and that $(0.170 \approx) 5 / \sqrt{864}<\tilde{\varepsilon}<1 / \sqrt{27}(\approx 0.192)$, which improves the lower bound of $\tilde{\varepsilon}$. Numerical simulation shows that $\tilde{\varepsilon} \approx 0.178$. 
For (1.1), when

$$
\varepsilon=1
$$

and $f_{\varepsilon=1}(u)$ has three real zeros $a<b<c$, in a celebrated paper [19, Section 2], Smoller and Wasserman first systematically studied bifurcation diagrams of positive solutions. In this case, problem (1.1) can be written as

$$
\left\{\begin{array}{l}
u^{\prime \prime}(x)+\lambda(u-a)(u-b)(c-u)=0, \quad-1<x<1 \\
u(-1)=u(1)=0, \quad a<b<c .
\end{array}\right.
$$

In particular, when

$$
a<b<0<c \text { and } \gamma_{\varepsilon}=(a+b+c) / 3>0
$$

(see Figure 1(i)), Smoller and Wasserman [19, p. 277, lines 18-19] stated that "This case is rather difficult, and requires some new estimates." For (1.5), (1.6), they [19, pp. 277-282] proved that, on the $\left(\lambda,\|u\|_{\infty}\right)$-plane,

(i) the bifurcation curve $S_{\varepsilon=1}$ is monotone increasing if $a b c \geq\left(\frac{a+b+c}{3}\right)^{3}$,

(ii) the bifurcation curve $S_{\varepsilon=1}$ is S-shaped if $a b c \leq \frac{16}{27}\left(\frac{a+b+c}{3}\right)^{3}$.

For the remaining case,

$$
\frac{16}{27}\left(\frac{a+b+c}{3}\right)^{3}<a b c<\left(\frac{a+b+c}{3}\right)^{3},
$$

Smoller and Wasserman left it as an open problem and conjectured that (1.5), (1.6) has at most three positive solutions for each $\lambda>0$; see [19, p. 283, lines 2-3]. In Theorem 2.5 stated below, we prove this conjecture.

Finally, in this section, we note that similar global bifurcation and exact multiplicity results of positive solutions have been studied by $\mathrm{Lu}$ [15, 16] for the positone problem

$$
\left\{\begin{array}{l}
u^{\prime \prime}(x)+\frac{1-u}{k \lambda^{2}\left[(1-u)^{2}+2 \varepsilon(1-u)+4 k \varepsilon^{2}\right]}=0, \quad-1<x<1, \\
u(-1)=u(1)=0, \quad k, \lambda, \varepsilon>0,
\end{array}\right.
$$

which arises from a mono-enzyme membrane model established in 1975 [10; see [16. Theorems 1-3] for the complete detailed results. To (1.8), Lu applied changes of variables and analysis techniques to give a rigorous proof. However, we have used different tools, the time-map techniques, to prove our main results.

The paper is organized as follows. Section 2 contains statements of main results. Section 3 contains several lemmas needed to prove the main results. Section 4 contains the proofs of the main results. Finally, in Section 5, we conclude this paper by giving a remark on the time-map techniques developed in this paper, and state three open problems with observed similar global bifurcation results in Theorem 2.1.

\section{MAin RESUlts}

The main results in this paper are Theorems 2.1 and 2.3-2.5 and Corollary 2.2. Note that Theorem 2.1 proves [23, Conjecture 1.3] with $f_{\varepsilon}(u)=1+u^{2}-\varepsilon u^{3}$. Corollary 2.2 follows immediately from Theorem 2.1, and consequently we omit its proof. 
Theorem 2.1. Consider (1.1), (1.2) with varying $\varepsilon>0$. There exists a positive number $\tilde{\varepsilon}=\tilde{\varepsilon}(\sigma, \rho, \tau)$ satisfying

such that

$$
\left(\frac{25}{32}\left(\frac{\sigma^{3}}{27 \rho}\right)\right)^{1 / 2}<\tilde{\varepsilon}<\left(\frac{\sigma^{3}}{27 \rho}\right)^{1 / 2}
$$

(i) (See Figure 2(i).) For $0<\varepsilon<\tilde{\varepsilon}$, the bifurcation curve $S_{\varepsilon}$ is $S$-shaped on the $\left(\lambda,\|u\|_{\infty}\right)$-plane. Moreover, there exist two positive numbers $\lambda_{*}<\lambda^{*}$ such that (1.1), (1.2) has exactly one degenerate positive solution $u_{\lambda_{*}}$ and $u_{\lambda^{*}}$ for $\lambda=\lambda_{*}$ and $\lambda=\lambda^{*}$, respectively. More precisely, (1.1), (1.2) has:

(a) exactly three positive solutions $u_{\lambda}, v_{\lambda}$, $w_{\lambda}$ with $w_{\lambda}<u_{\lambda}<v_{\lambda}$ for $\lambda_{*}<\lambda<\lambda^{*}$,

(b) exactly two positive solutions $w_{\lambda}$, $u_{\lambda}$ with $w_{\lambda}<u_{\lambda}$ for $\lambda=\lambda_{*}$, and exactly two positive solutions $u_{\lambda}, v_{\lambda}$ with $u_{\lambda}<v_{\lambda}$ for $\lambda=\lambda^{*}$,

(c) exactly one positive solution $w_{\lambda}$ for $0<\lambda<\lambda_{*}$, and exactly one positive solution $v_{\lambda}$ for $\lambda>\lambda^{*}$.

Furthermore,

(d) $\lim _{\lambda \rightarrow 0^{+}}\left\|w_{\lambda}\right\|_{\infty}=0$ and $\lim _{\lambda \rightarrow \infty}\left\|v_{\lambda}\right\|_{\infty}=\beta_{\varepsilon}$.

(ii) (See Figure 2(ii).) For $\varepsilon=\tilde{\varepsilon}$, the bifurcation curve $S_{\tilde{\varepsilon}}$ is monotone increasing on the $\left(\lambda,\|u\|_{\infty}\right)$-plane. Moreover, (1.1), (1.2) has exactly one (cusp type) degenerate positive solution $u_{\tilde{\lambda}}$. More precisely, for all $\lambda>0$, (1.1), (1.2) has exactly one positive solution $u_{\lambda}$ satisfying $\lim _{\lambda \rightarrow 0^{+}}\left\|u_{\lambda}\right\|_{\infty}=0$ and $\lim _{\lambda \rightarrow \infty}\left\|u_{\lambda}\right\|_{\infty}=\beta_{\varepsilon}$.

(iii) (See Figure 2(iii).) For $\varepsilon>\tilde{\varepsilon}$, the bifurcation curve $S_{\varepsilon}$ is monotone increasing on the $\left(\lambda,\|u\|_{\infty}\right)$-plane. Moreover, all positive solutions $u_{\lambda}$ of (1.1), (1.2) are nondegenerate. More precisely, for all $\lambda>0$, (1.1), (1.2) has exactly one positive solution $u_{\lambda}$ satisfying $\lim _{\lambda \rightarrow 0^{+}}\left\|u_{\lambda}\right\|_{\infty}=0$ and $\lim _{\lambda \rightarrow \infty}\left\|u_{\lambda}\right\|_{\infty}=\beta_{\varepsilon}$.

Corollary 2.2. Consider (1.3) with varying $\varepsilon>0$. There exists a positive number $\tilde{\varepsilon}$ satisfying

such that

$$
(0.170 \approx) \frac{5}{\sqrt{864}}<\tilde{\varepsilon}<\frac{1}{\sqrt{27}}(\approx 0.192)
$$

(i) (See Figure 2(i).) For $0<\varepsilon<\tilde{\varepsilon}$, the bifurcation curve $S_{\varepsilon}$ is $S$-shaped on the $\left(\lambda,\|u\|_{\infty}\right)$-plane. Moreover, there exist two positive numbers $\lambda_{*}<\lambda^{*}$ such that (1.3) has exactly one degenerate positive solution $u_{\lambda_{*}}$ and $u_{\lambda^{*}}$ for $\lambda=\lambda_{*}$ and $\lambda=\lambda^{*}$, respectively. More precisely, problem (1.3) has:

(a) exactly three positive solutions $u_{\lambda}, v_{\lambda}, w_{\lambda}$ with $w_{\lambda}<u_{\lambda}<v_{\lambda}$ for $\lambda_{*}<\lambda<\lambda^{*}$,

(b) exactly two positive solutions $w_{\lambda}$, $u_{\lambda}$ with $w_{\lambda}<u_{\lambda}$ for $\lambda=\lambda_{*}$, and exactly two positive solutions $u_{\lambda}, v_{\lambda}$ with $u_{\lambda}<v_{\lambda}$ for $\lambda=\lambda^{*}$,

(c) exactly one positive solution $w_{\lambda}$ for $0<\lambda<\lambda_{*}$, and exactly one positive solution $v_{\lambda}$ for $\lambda>\lambda^{*}$.

Furthermore,

(d) $\lim _{\lambda \rightarrow 0^{+}}\left\|w_{\lambda}\right\|_{\infty}=0$ and $\lim _{\lambda \rightarrow \infty}\left\|v_{\lambda}\right\|_{\infty}=\beta_{\varepsilon}$.

(ii) (See Figure 2(ii).) For $\varepsilon=\tilde{\varepsilon}$, the bifurcation curve $S_{\tilde{\varepsilon}}$ is monotone increasing on the $\left(\lambda,\|u\|_{\infty}\right)$-plane. Moreover, problem (1.3) has exactly one (cusp type) degenerate positive solution $u_{\tilde{\lambda}}$. More precisely, for all $\lambda>0$, problem 
(1.3) has exactly one positive solution $u_{\lambda}$ satisfying $\lim _{\lambda \rightarrow 0^{+}}\left\|u_{\lambda}\right\|_{\infty}=0$ and $\lim _{\lambda \rightarrow \infty}\left\|u_{\lambda}\right\|_{\infty}=\beta_{\varepsilon}$.

(iii) (See Figure 2(iii).) For $\varepsilon>\tilde{\varepsilon}$, the bifurcation curve $S_{\varepsilon}$ is monotone increasing on the $\left(\lambda,\|u\|_{\infty}\right)$-plane. Moreover, all positive solutions $u_{\lambda}$ of (1.3) are nondegenerate. More precisely, for all $\lambda>0$, problem (1.3) has exactly one positive solution $u_{\lambda}$ satisfying $\lim _{\lambda \rightarrow 0^{+}}\left\|u_{\lambda}\right\|_{\infty}=0$ and $\lim _{\lambda \rightarrow \infty}\left\|u_{\lambda}\right\|_{\infty}=$ $\beta_{\varepsilon}$.

For any $\lambda>0$, on the $\left(\varepsilon,\|u\|_{\infty}\right)$-plane, it is interesting to study the shape and structure of bifurcation curves $\Sigma_{\lambda}$ of positive solutions of (1.1), (1.2), defined by

$$
\Sigma_{\lambda} \equiv\left\{\left(\varepsilon,\left\|u_{\varepsilon}\right\|_{\infty}\right): \varepsilon>0 \text { and } u_{\varepsilon} \text { is a positive solution of (1.1), (1.2) }\right\} \text {. }
$$

(Note that we allow that the bifurcation curve $\Sigma_{\lambda}$ consists of two (or more) connected components.) We say that, on the $\left(\varepsilon,\|u\|_{\infty}\right)$-plane, the bifurcation curve $\Sigma_{\lambda}$ is reversed S-shaped if $\Sigma_{\lambda}$ is a continuous curve and there exist two numbers $\varepsilon_{*}<\varepsilon^{*}$ such that $\Sigma_{\lambda}$ has exactly two turning points at some points $\left(\varepsilon_{*},\left\|u_{\varepsilon_{*}}\right\|_{\infty}\right)$ and $\left(\varepsilon^{*},\left\|u_{\varepsilon^{*}}\right\|_{\infty}\right)$, and

(i) $\varepsilon_{*}<\varepsilon^{*}$ and $\left\|u_{\varepsilon_{*}}\right\|_{\infty}<\left\|u_{\varepsilon^{*}}\right\|_{\infty}$,

(ii) at $\left(\varepsilon_{*},\left\|u_{\varepsilon_{*}}\right\|_{\infty}\right)$ the bifurcation curve $\Sigma_{\lambda}$ turns to the right,

(iii) at $\left(\varepsilon^{*},\left\|u_{\varepsilon^{*}}\right\|_{\infty}\right)$ the bifurcation curve $\Sigma_{\lambda}$ turns to the left.

See Figure 3(iii) for example.

In the next theorem for (1.1), (1.2) with varying $\lambda>0$, we prove the global bifurcation of bifurcation curves $\Sigma_{\lambda}$ and determine the exact multiplicity of positive solutions by the values of $\lambda$ and $\varepsilon$; see Figure 3. See also Figure 4 depicted below.

Theorem 2.3. Consider (1.1), (1.2) with varying $\lambda>0$. There exist two positive numbers $\lambda_{0}\left(=\lambda_{0}(\sigma, \rho, \tau)\right)<\tilde{\lambda}(=\tilde{\lambda}(\sigma, \rho, \tau))$ such that

(i) (See Figure 3(i).) For $0<\lambda<\lambda_{0}$, on the $\left(\varepsilon,\|u\|_{\infty}\right)$-plane, the bifurcation curve $\Sigma_{\lambda}$ has two disjoint connected components, the upper branch is つshaped with exactly one turning point, and the lower branch is a monotone decreasing curve. Moreover, there exists a positive number $\varepsilon^{*}$ such that (1.1), (1.2) has exactly one degenerate positive solution $u_{\varepsilon^{*}}$ for $\varepsilon=\varepsilon^{*}$. More precisely, problem (1.1), (1.2) has:

(a) exactly three positive solutions $u_{\varepsilon}, v_{\varepsilon}, w_{\varepsilon}$ with $w_{\varepsilon}<u_{\varepsilon}<v_{\varepsilon}$ for $0<$ $\varepsilon<\varepsilon^{*}$,

(b) exactly two positive solutions $w_{\varepsilon}, u_{\varepsilon}$ with $w_{\varepsilon}<u_{\varepsilon}$ for $\varepsilon=\varepsilon^{*}$,

(c) exactly one positive solution $w_{\varepsilon}$ for $\varepsilon>\varepsilon^{*}$.

Furthermore,

(d) $0=\lim _{\varepsilon \rightarrow \infty}\left\|w_{\varepsilon}\right\|_{\infty}<\lim _{\varepsilon \rightarrow 0^{+}}\left\|w_{\varepsilon}\right\|_{\infty}<\lim _{\varepsilon \rightarrow 0^{+}}\left\|u_{\varepsilon}\right\|_{\infty}<$ $\lim _{\varepsilon \rightarrow 0^{+}}\left\|v_{\varepsilon}\right\|_{\infty}=\infty$.

(ii) (See Figure 3(ii).) For $\lambda=\lambda_{0}$, on the $\left(\varepsilon,\|u\|_{\infty}\right)$-plane, the bifurcation curve $\Sigma_{\lambda_{0}}$ has two disjoint connected components: the upper branch is $\supset$ shaped with exactly one turning point, and the lower branch is a monotone decreasing curve. Moreover, there exists a positive number $\varepsilon^{*}$ such that (1.1), (1.2) has exactly one degenerate positive solution $u_{\varepsilon^{*}}$ for $\varepsilon=\varepsilon^{*}$. More precisely, problem (1.1), (1.2) has:

(a) exactly three positive solutions $u_{\varepsilon}, v_{\varepsilon}, w_{\varepsilon}$ with $w_{\varepsilon}<u_{\varepsilon}<v_{\varepsilon}$ for $0<$ $\varepsilon<\varepsilon^{*}$

(b) exactly two positive solutions $w_{\varepsilon}, u_{\varepsilon}$ with $w_{\varepsilon}<u_{\varepsilon}$ for $\varepsilon=\varepsilon^{*}$, 


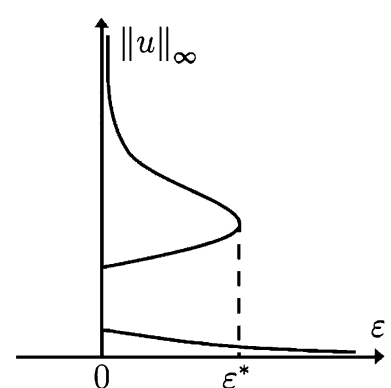

(i) $0<\lambda<\lambda_{0}$

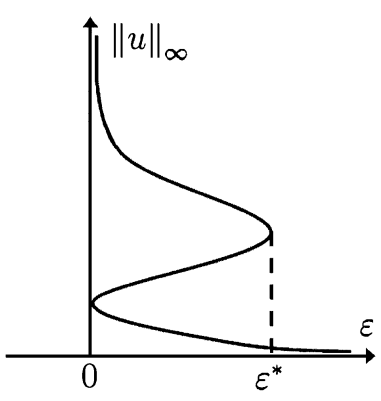

(ii) $\lambda=\lambda_{0}$

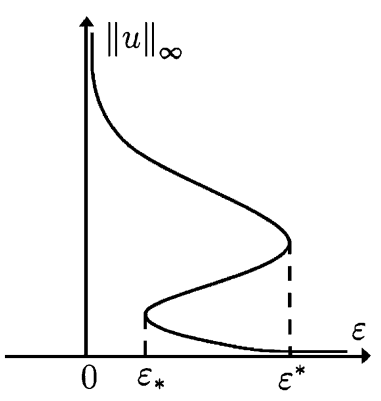

(iii) $\lambda_{0}<\lambda<\tilde{\lambda}$

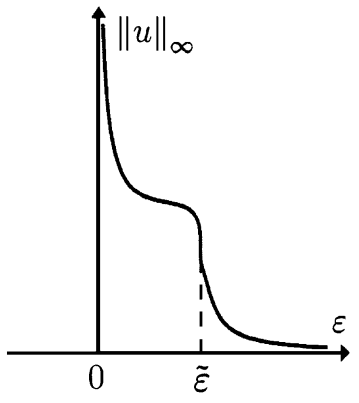

(iv) $\lambda=\tilde{\lambda}$

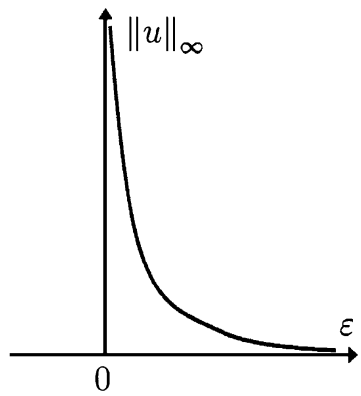

(v) $\lambda>\tilde{\lambda}$

Figure 3. Global bifurcation of bifurcation curves $\Sigma_{\lambda}$ of (1.1), (1.2) with varying $\lambda>0$.

(c) exactly one positive solution $w_{\varepsilon}$ for $\varepsilon>\varepsilon^{*}$.

Furthermore,

(d) $0=\lim _{\varepsilon \rightarrow \infty}\left\|w_{\varepsilon}\right\|_{\infty}<\lim _{\varepsilon \rightarrow 0^{+}}\left\|w_{\varepsilon}\right\|_{\infty}=\lim _{\varepsilon \rightarrow 0^{+}}\left\|u_{\varepsilon}\right\|_{\infty}<$ $\lim _{\varepsilon \rightarrow 0^{+}}\left\|v_{\varepsilon}\right\|_{\infty}=\infty$.

(iii) (See Figure 3(iii).) For $\lambda_{0}<\lambda<\tilde{\lambda}$, the bifurcation curve $\Sigma_{\lambda}$ is reversed $S$ shaped on the $\left(\varepsilon,\|u\|_{\infty}\right)$-plane. Moreover, there exist two positive numbers $\varepsilon_{*}<\varepsilon^{*}$ such that (1.1), (1.2) has exactly one degenerate positive solution $u_{\varepsilon_{*}}$ and $u_{\varepsilon^{*}}$ for $\varepsilon=\varepsilon_{*}$ and $\varepsilon=\varepsilon^{*}$, respectively. More precisely, problem (1.1), (1.2) has:

(a) exactly three positive solutions $u_{\varepsilon}, v_{\varepsilon}, w_{\varepsilon}$ with $w_{\varepsilon}<u_{\varepsilon}<v_{\varepsilon}$ for $\varepsilon_{*}<$ $\varepsilon<\varepsilon^{*}$

(b) exactly two positive solutions $u_{\varepsilon}$, $v_{\varepsilon}$ with $u_{\varepsilon}<v_{\varepsilon}$ for $\varepsilon=\varepsilon_{*}$, and exactly two positive solutions $w_{\varepsilon}, u_{\varepsilon}$ with $w_{\varepsilon}<u_{\varepsilon}$ for $\varepsilon=\varepsilon^{*}$,

(c) exactly one positive solution $v_{\varepsilon}$ for $0<\varepsilon<\varepsilon_{*}$, and exactly one positive solution $w_{\varepsilon}$ for $\varepsilon>\varepsilon^{*}$.

Furthermore,

(d) $\lim _{\varepsilon \rightarrow 0^{+}}\left\|v_{\varepsilon}\right\|_{\infty}=\infty$ and $\lim _{\varepsilon \rightarrow \infty}\left\|w_{\varepsilon}\right\|_{\infty}=0$.

(iv) (See Figure 3(iv).) For $\lambda=\tilde{\lambda}$, the bifurcation curve $\Sigma_{\tilde{\lambda}}$ is monotone decreasing on the $\left(\varepsilon,\|u\|_{\infty}\right)$-plane. Moreover, problem (1.1), (1.2) has exactly one (cusp type) degenerate positive solution $u_{\tilde{\varepsilon}}$. More precisely, for all 


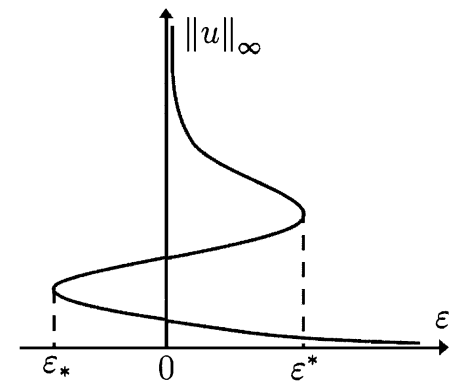

(i) $0<\lambda<\lambda_{0}$

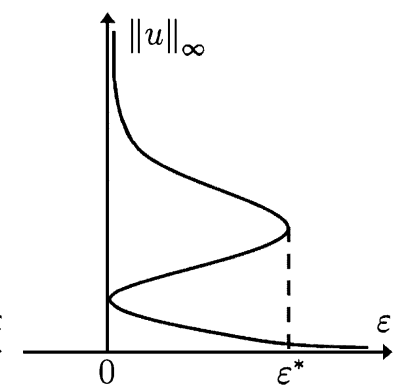

(ii) $\lambda=\lambda_{0}$

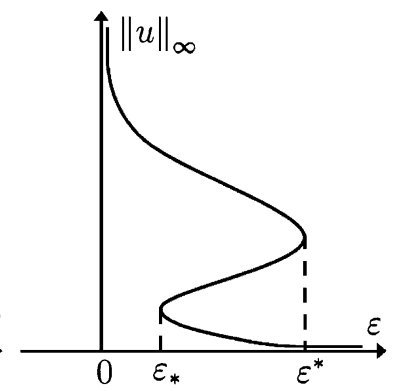

(iii) $\lambda_{0}<\lambda<\tilde{\lambda}$

Figure 4. Global bifurcation of bifurcation curves $\tilde{\Sigma}_{\lambda}$ of (1.1), (1.2) with $\varepsilon>0$ generalized to $\varepsilon \in \mathbb{R}$ and with varying $\lambda \in(0, \tilde{\lambda})$.

$\varepsilon>0$, problem (1.1), (1.2) has exactly one positive solution $u_{\varepsilon}$ satisfying $\lim _{\varepsilon \rightarrow 0^{+}}\left\|u_{\varepsilon}\right\|_{\infty}=\infty$ and $\lim _{\varepsilon \rightarrow \infty}\left\|u_{\varepsilon}\right\|_{\infty}=0$.

(v) (See Figure 3(v).) For $\lambda>\tilde{\lambda}$, the bifurcation curve $\Sigma_{\lambda}$ is monotone decreasing on the $\left(\varepsilon,\|u\|_{\infty}\right)$-plane. Moreover, all positive solutions $u_{\varepsilon}$ of (1.1), (1.2) are nondegenerate. More precisely, for all $\varepsilon>0$, problem (1.1), (1.2) has exactly one positive solution $u_{\varepsilon}$ satisfying $\lim _{\varepsilon \rightarrow 0^{+}}\left\|u_{\varepsilon}\right\|_{\infty}=\infty$ and $\lim _{\varepsilon \rightarrow \infty}\left\|u_{\varepsilon}\right\|_{\infty}=0$.

We give the next remark to Theorem 2.3.

Remark 2.1 (Cf. 23, Theorem 2.3]). Considering (1.1), (1.2) with $\varepsilon>0$ generalized to $\varepsilon \in \mathbb{R}$, we define the bifurcation curve

$$
\tilde{\Sigma}_{\lambda} \equiv\left\{\left(\varepsilon,\left\|u_{\varepsilon}\right\|_{\infty}\right): \varepsilon \in \mathbb{R} \text { and } u_{\varepsilon} \text { is a positive solution of (1.1), (1.2) }\right\} \text {. }
$$

Actually, it can be easily proved that:

(i) For $0<\lambda<\lambda_{0}$, the bifurcation curve $\tilde{\Sigma}_{\lambda}$ is reversed S-shaped on the $\left(\varepsilon,\|u\|_{\infty}\right)$-plane. Moreover, there exists $\varepsilon_{*}<0$ such that (1.1), (1.2) has exactly two positive solutions $w_{\varepsilon}, u_{\varepsilon}$ with $w_{\varepsilon}<u_{\varepsilon}$ for $\varepsilon_{*}<\varepsilon \leq 0$, and exactly one positive solution $u_{\varepsilon}$ for $\varepsilon=\varepsilon_{*}$, and no positive solution for $\varepsilon<\varepsilon_{*}$. See Figure 4(i).

(ii) For $\lambda=\lambda_{0}$, the bifurcation curve $\tilde{\Sigma}_{\lambda_{0}}$ is reversed S-shaped on the $\left(\varepsilon,\|u\|_{\infty}\right)$ plane. Moreover, problem (1.1), (1.2) has exactly one positive solution $u_{\varepsilon}$ for $\varepsilon=0$, and no positive solution for $\varepsilon<0$. See Figure 4(ii).

Notice that, by Theorem 2.1, on the $\left(\lambda,\|u\|_{\infty}\right)$-plane, the bifurcation curve $S_{\varepsilon}$ is S-shaped for $0<\varepsilon<\tilde{\varepsilon}$; see Figure 2. While by Theorem 2.3 and Remark 2.1, on the $\left(\varepsilon,\|u\|_{\infty}\right)$-plane, the bifurcation curve $\tilde{\Sigma}_{\lambda}$ is reversed S-shaped for $0<\lambda<\tilde{\lambda}$; see Figure 4.

We study, in the $\left(\varepsilon, \lambda,\|u\|_{\infty}\right)$-space, the shape and structure of the bifurcation surface $\Gamma$ of positive solutions of (1.1), (1.2), defined by

$$
\Gamma \equiv\left\{\left(\varepsilon, \lambda,\left\|u_{\varepsilon, \lambda}\right\|_{\infty}\right): \varepsilon, \lambda>0 \text { and } u_{\varepsilon, \lambda} \text { is a positive solution of (1.1), (1.2) }\right\} \text {, }
$$




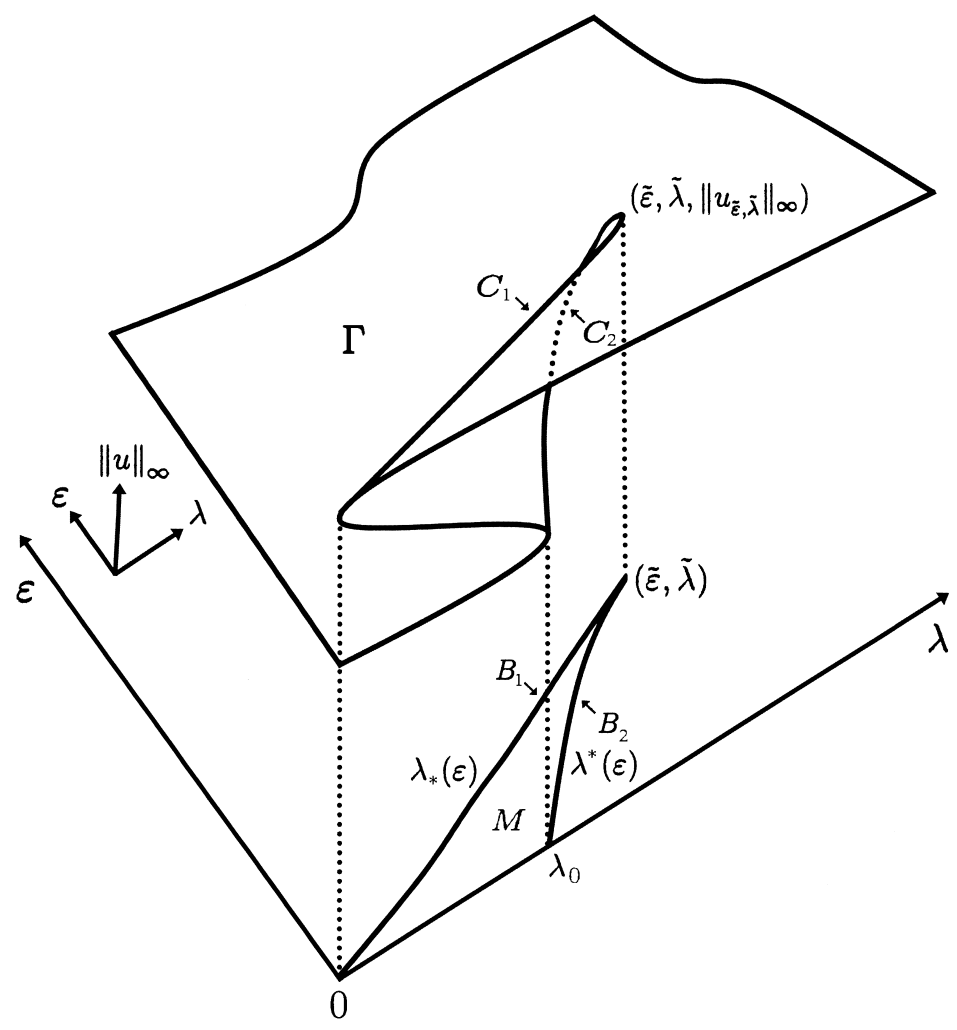

Figure 5. The bifurcation surface $\Gamma$ of (1.1), (1.2) with the fold curve $C_{\Gamma}=C_{1} \cup C_{2}$, and the projection of $\Gamma$ onto $F_{q} . B_{\Gamma}=B_{1} \cup B_{2}$ is the bifurcation set and $(\tilde{\varepsilon}, \tilde{\lambda})$ is the cusp point on $F_{q}$.

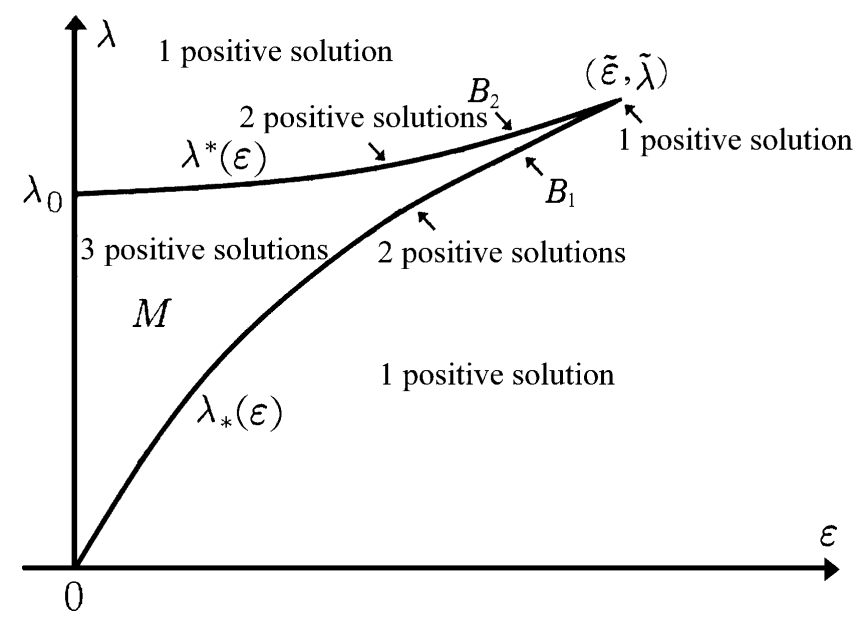

Figure 6 . The projection of the bifurcation surface $\Gamma$ onto $F_{q}$. $B_{\Gamma}=B_{1} \cup B_{2}$ is the bifurcation set and $(\tilde{\varepsilon}, \tilde{\lambda})$ is the cusp point on $F_{q}$. 
which has the appearance of a folded surface with the fold curve

$$
C_{\Gamma} \equiv\left\{\left(\varepsilon, \lambda,\left\|u_{\varepsilon, \lambda}\right\|_{\infty}\right): \varepsilon, \lambda>0 \text { and } u_{\varepsilon, \lambda}\right. \text { is a degenerate positive solution of }
$$

(1.1), (1.2)\}.

Let $F_{q}$ denote the first quadrant of the $(\varepsilon, \lambda)$-parameter plane. We also study, on $F_{q}$, the bifurcation set

$B_{\Gamma} \equiv\left\{(\varepsilon, \lambda): \varepsilon, \lambda>0\right.$ and $u_{\varepsilon, \lambda}$ is a degenerate positive solution of (1.1), (1.2) $\}$,

which is the projection of the fold curve $C_{\Gamma}$ onto $F_{q}$. Let $M$ denote the bounded, open connected subset of $F_{q}$, which is 'inside' $B_{\Gamma}$. See Figures 5 and 6 .

Let $\tilde{\varepsilon}=\tilde{\varepsilon}(\sigma, \rho, \tau), \lambda_{0}=\lambda_{0}(\sigma, \rho, \tau), \tilde{\lambda}=\tilde{\lambda}(\sigma, \rho, \tau), \lambda_{*}=\lambda_{*}(\varepsilon), \lambda^{*}=\lambda^{*}(\varepsilon)$, $\varepsilon_{*}=\varepsilon_{*}(\lambda)$ and $\varepsilon^{*}=\varepsilon^{*}(\lambda)$ be the values in Theorems 2.1 and 2.3 for (1.1), (1.2) with $\varepsilon>0$. We let curves

$$
B_{1} \equiv\left\{\left(\varepsilon, \lambda_{*}(\varepsilon)\right): 0<\varepsilon \leq \tilde{\varepsilon}\right\} \text { and } B_{2} \equiv\left\{\left(\varepsilon, \lambda^{*}(\varepsilon)\right): 0<\varepsilon \leq \tilde{\varepsilon}\right\},
$$

and we study the structure of the bifurcation set $B_{\Gamma}$ in the next theorem.

Theorem 2.4 (See Figure 6). Consider (1.1), (1.2) with $(\varepsilon, \lambda) \in F_{q}$. Then the bifurcation set $B_{\Gamma}=B_{1} \cup B_{2}$. Moreover, problem (1.1), (1.2) has exactly two positive solutions for $(\varepsilon, \lambda) \in B_{\Gamma} \backslash\{(\tilde{\varepsilon}, \tilde{\lambda})\}$, exactly three positive solutions for $(\varepsilon, \lambda) \in M$, and exactly one positive solution for $(\varepsilon, \lambda) \in\left(F_{q} \backslash\left(B_{\Gamma} \cup M\right)\right) \cup\{(\tilde{\varepsilon}, \tilde{\lambda})\}$. More precisely,

(i) Functions $\lambda_{*}(\varepsilon)$ and $\lambda^{*}(\varepsilon)$ are both continuous, strictly increasing on $(0, \tilde{\varepsilon}]$ and satisfy $0=\lim _{\varepsilon \rightarrow 0^{+}} \lambda_{*}(\varepsilon)<\lim _{\varepsilon \rightarrow 0^{+}} \lambda^{*}(\varepsilon)=\lambda_{0}<\tilde{\lambda}=\lambda_{*}(\tilde{\varepsilon})=\lambda^{*}(\tilde{\varepsilon})$.

(ii) Function $\varepsilon^{*}(\lambda)$ is continuous, strictly increasing on $(0, \tilde{\lambda}]$ and satisfies $\lim _{\lambda \rightarrow 0^{+}} \varepsilon^{*}(\lambda)=0$ and $\varepsilon^{*}(\tilde{\lambda})=\tilde{\varepsilon}$. Function $\varepsilon_{*}(\lambda)$ is continuous, strictly increasing on $\left(\lambda_{0}, \tilde{\lambda}\right]$ and satisfies $\lim _{\lambda \rightarrow \lambda_{0}^{+}} \varepsilon_{*}(\lambda)=0$ and $\varepsilon_{*}(\tilde{\lambda})=\tilde{\varepsilon}$.

In the next remark, we give a precise characterization of the fold curve $C_{\Gamma}$ in the $\left(\varepsilon, \lambda,\|u\|_{\infty}\right)$-space.

Remark 2.2 (See Figure 5). Consider (1.1), (1.2). Then, by Theorem 2.4(i), the fold curve $C_{\Gamma}=C_{1} \cup C_{2}$, where

$$
C_{1} \equiv\left\{\left(\varepsilon, \lambda_{*}(\varepsilon),\left\|u_{\varepsilon, \lambda_{*}(\varepsilon)}\right\|_{\infty}\right): 0<\varepsilon \leq \tilde{\varepsilon}\right\}
$$

and

$$
C_{2} \equiv\left\{\left(\varepsilon, \lambda^{*}(\varepsilon),\left\|u_{\varepsilon, \lambda^{*}(\varepsilon)}\right\|_{\infty}\right): 0<\varepsilon \leq \tilde{\varepsilon}\right\} .
$$

Moreover, by applying (4.4)-4.7) stated below, we can prove that:

(i) $\left\|u_{\varepsilon, \lambda_{*}(\varepsilon)}\right\|_{\infty}>\left\|u_{\varepsilon, \lambda^{*}(\varepsilon)}\right\|_{\infty}$ for $0<\varepsilon<\tilde{\varepsilon}$ and $\left\|u_{\tilde{\varepsilon}, \lambda_{*}(\tilde{\varepsilon})}\right\|_{\infty}=\left\|u_{\tilde{\varepsilon}, \lambda^{*}(\tilde{\varepsilon})}\right\|_{\infty}=$ $\left\|u_{\tilde{\varepsilon}, \tilde{\lambda}}\right\|_{\infty}$.

(ii) $\left\|u_{\varepsilon, \lambda_{*}(\varepsilon)}\right\|_{\infty}$ is a continuous, strictly decreasing function of $\varepsilon \in(0, \tilde{\varepsilon}]$ and $\left\|u_{\varepsilon, \lambda^{*}(\varepsilon)}\right\|_{\infty}^{\infty}$ is a continuous, strictly increasing function of $\varepsilon \in(0, \tilde{\varepsilon}]$.

(iii) $C_{\Gamma}$ is a continuous curve in the $\left(\varepsilon, \lambda,\|u\|_{\infty}\right)$-space.

In particular, for (1.3) with $\sigma=\tau=\rho=1$, the numerical simulation shows that $\left(\tilde{\varepsilon}, \tilde{\lambda},\left\|u_{\tilde{\varepsilon}, \tilde{\lambda}}\right\|_{\infty}\right) \approx(0.178,0.881,2.113)$.

Observe that $\lambda^{*}(\varepsilon)$ and $\lambda_{*}(\varepsilon)$ both have continuous inverse functions on $(0, \tilde{\varepsilon}]$. Indeed, $\varepsilon_{*}(\lambda)$ is the inverse function of $\lambda^{*}(\varepsilon)$ on $\left(\lambda_{0}, \tilde{\lambda}\right]$, and $\varepsilon^{*}(\lambda)$ is the inverse function of $\lambda_{*}(\varepsilon)$ on $(0, \tilde{\lambda}]$, respectively. 
Theorem 2.5. Consider (1.5), (1.6). Suppose that (1.7) holds. Then:

(i) (See Figure 2(i).) For

$$
\frac{16}{27}\left(\frac{a+b+c}{3}\right)^{3}<a b c \leq \frac{25}{32}\left(\frac{a+b+c}{3}\right)^{3},
$$

the bifurcation curve $S_{\varepsilon=1}$ is $S$-shaped on the $\left(\lambda,\|u\|_{\infty}\right)$-plane. Moreover, there exist two positive numbers $\lambda_{*}<\lambda^{*}$ such that (1.5), (1.6) has exactly two degenerate positive solutions $u_{\lambda_{*}}$ and $u_{\lambda^{*}}$ for $\lambda=\lambda_{*}$ and $\lambda=\lambda^{*}$, respectively. More precisely, problem (1.5), (1.6) has:

(a) exactly three positive solutions $u_{\lambda}, v_{\lambda}, w_{\lambda}$ with $w_{\lambda}<u_{\lambda}<v_{\lambda}$ for $\lambda_{*}<\lambda<\lambda^{*}$,

(b) exactly two positive solutions $w_{\lambda}$, $u_{\lambda}$ with $w_{\lambda}<u_{\lambda}$ for $\lambda=\lambda_{*}$, and exactly two positive solutions $u_{\lambda}, v_{\lambda}$ with $u_{\lambda}<v_{\lambda}$ for $\lambda=\lambda^{*}$,

(c) exactly one positive solution $w_{\lambda}$ for $0<\lambda<\lambda_{*}$, and exactly one positive solution $v_{\lambda}$ for $\lambda>\lambda^{*}$.

Furthermore,

(d) $\lim _{\lambda \rightarrow 0^{+}}\left\|w_{\lambda}\right\|_{\infty}=0$ and $\lim _{\lambda \rightarrow \infty}\left\|v_{\lambda}\right\|_{\infty}=c$.

(ii) (See Figure 2(i)-(iii) and Theorem 2.1(i)-(iii).) For

$$
\frac{25}{32}\left(\frac{a+b+c}{3}\right)^{3}<a b c<\left(\frac{a+b+c}{3}\right)^{3},
$$

the bifurcation curve $S_{\varepsilon=1}$ is either $S$-shaped or monotone increasing on the $\left(\lambda,\|u\|_{\infty}\right)$-plane. Moreover, problem (1.5), (1.6) has at most three positive solutions for each $\lambda>0$.

\section{LEMMAS}

In this section, in the next Lemmas 3.1-3.9, we develop new time-map techniques to prove Theorem 2.1 for (1.1), (1.2). In particular, Lemma 3.3 is a key lemma in the proof of Theorem 2.1, in which, for any fixed $\varepsilon>0$, we prove that the bifurcation curve $S_{\varepsilon}$ is either monotone increasing or S-shaped on the $\left(\lambda,\|u\|_{\infty}\right)$-plane. To apply the time-map techniques for (1.1), (1.2), in the following, we consider $\varepsilon \geq 0$. The time-map formula which we apply to study (1.1), (1.2) takes the form:

$$
\sqrt{\lambda}=\frac{1}{\sqrt{2}} \int_{0}^{\alpha}\left[F_{\varepsilon}(\alpha)-F_{\varepsilon}(u)\right]^{-1 / 2} d u \equiv T_{\varepsilon}(\alpha) \text { for } 0<\alpha<\beta_{\varepsilon} \text { if } \varepsilon \geq 0,
$$

where $F_{\varepsilon}(u) \equiv \int_{0}^{u} f_{\varepsilon}(t) d t$ and $\beta_{\varepsilon}$ is the unique positive zero of the cubic polynomial $f_{\varepsilon}(u)$ for $\varepsilon>0$, and $\beta_{\varepsilon=0} \equiv \infty$; see Laetsch [13]. So positive solutions $u_{\varepsilon, \lambda}$ for (1.1), (1.2) correspond to $\left\|u_{\varepsilon, \lambda}\right\|_{\infty}=\alpha$ and $T_{\varepsilon}(\alpha)=\sqrt{\lambda}$. Thus, studying of the exact number of positive solutions of (1.1), (1.2) for fixed $\varepsilon \geq 0$ is equivalent to studying the shape of the time-map $T_{\varepsilon}(\alpha)$ on $\left(0, \beta_{\varepsilon}\right)$; studying the exact number of positive solutions of (1.1), (1.2) for fixed $\lambda>0$ is equivalent to studying the number of roots of the equation $T_{\varepsilon}(\alpha)=\sqrt{\lambda}$ on $\left(0, \beta_{\varepsilon}\right)$ for varying $\varepsilon>0$. Note that it can be proved that $T_{\varepsilon}(\alpha)$ is a thrice differentiable function of $\alpha \in\left(0, \beta_{\varepsilon}\right)$ for $\varepsilon \geq 0$. The proof is easy but tedious and we omit it.

We say that a positive solution $u_{\varepsilon, \lambda}$ of (1.1), (1.2) is degenerate if $T_{\varepsilon}^{\prime}\left(\left\|u_{\varepsilon, \lambda}\right\|_{\infty}\right)=$ 0 and is nondegenerate if $T_{\varepsilon}^{\prime}\left(\left\|u_{\varepsilon, \lambda}\right\|_{\infty}\right) \neq 0$. So to find the degenerate positive solutions of (1.1), (1.2), we only need to find the critical points of $T_{\varepsilon}(\alpha)$ on $\left(0, \beta_{\varepsilon}\right)$. It is known that a degenerate positive solution $u_{\varepsilon, \lambda}$ of (1.1), (1.2) is of cusp type if $T_{\varepsilon}^{\prime \prime}\left(\left\|u_{\varepsilon, \lambda}\right\|_{\infty}\right)=0$ and $T_{\varepsilon}^{\prime \prime \prime}\left(\left\|u_{\varepsilon, \lambda}\right\|_{\infty}\right) \neq 0$; see Shi [18, p. 214] and [17, p. 497]. 
The main difficulty to obtain our result is to obtain the exact number of critical points of the time-map $T_{\varepsilon}(\alpha)$ on $\left(0, \beta_{\varepsilon}\right)$ for all $\varepsilon>0$. This question is partially answered in Lemmas 3.1 and 3.2. Lemma 3.1 follows from [13, Theorems 2.6, 2.9 and 3.2], and Lemma 3.2 mainly follows by applying [11, Theorem 2.1] or from [21, Theorem 3]. We omit the proofs.

Lemma 3.1. Consider (1.1), (1.2). For any fixed $\varepsilon>0$,

(i) $\lim _{\alpha \rightarrow 0^{+}} T_{\varepsilon}(\alpha)=0$ and $\lim _{\alpha \rightarrow \beta_{\varepsilon}^{-}} T_{\varepsilon}(\alpha)=\infty$.

(ii) If $T_{\varepsilon}(\alpha)$ is not strictly increasing on $\left(0, \gamma_{\varepsilon}\right)$, then $T_{\varepsilon}(\alpha)$ is strictly increasing on $\left(0, \tilde{\gamma}_{\varepsilon}\right)$ and strictly decreasing on $\left(\tilde{\gamma}_{\varepsilon}, \gamma_{\varepsilon}\right)$ for some $\tilde{\gamma}_{\varepsilon} \in\left(0, \gamma_{\varepsilon}\right)$.

Lemma 3.2. Consider (1.1), (1.2). Then:

(i) For any fixed $\varepsilon \geq\left(\frac{\sigma^{3}}{27 \rho}\right)^{1 / 2}, T_{\varepsilon}(\alpha)$ is a strictly increasing function on $\left(0, \beta_{\varepsilon}\right)$.

(ii) For any fixed positive $\varepsilon \leq\left(\frac{1}{2}\left(\frac{\sigma^{3}}{27 \rho}\right)\right)^{1 / 2}, T_{\varepsilon}(\alpha)$ has exactly one local maximum and one local minimum on $\left(0, \beta_{\varepsilon}\right)$.

However, there is a gap, what about the case where $\varepsilon$ is between $\left(\frac{1}{2}\left(\frac{\sigma^{3}}{27 \rho}\right)\right)^{1 / 2}$ and $\left(\frac{\sigma^{3}}{27 \rho}\right)^{1 / 2}$ ? First, in Lemma 3.3, we prove

Lemma 3.3. Consider (1.1), (1.2). For any fixed $\varepsilon>0, T_{\varepsilon}(\alpha)$ is either a strictly increasing function or has exactly two critical points, a local maximum and a local minimum, on $\left(0, \beta_{\varepsilon}\right)$.

To prove Lemma 3.3, we develop some new time-map techniques. First, for the time-map function $T_{\varepsilon}(\alpha)$ with $\alpha \in\left(0, \beta_{\varepsilon}\right)$ in (3.1), letting $u=\alpha v$, we have

$$
T_{\varepsilon}(\alpha)=\frac{\alpha}{\sqrt{2}} \int_{0}^{1} \frac{1}{\left[F_{\varepsilon}(\alpha)-F_{\varepsilon}(\alpha v)\right]^{1 / 2}} d v .
$$

For any fixed $\varepsilon>0$, we define the auxiliary function

$$
\begin{aligned}
H_{\varepsilon}(\alpha)= & 12 \sqrt{2} T_{\varepsilon}^{\prime}(\alpha)+8 \sqrt{2} \alpha T_{\varepsilon}^{\prime \prime}(\alpha) \\
= & 12 \int_{0}^{1} \frac{1}{\left[F_{\varepsilon}(\alpha)-F_{\varepsilon}(\alpha v)\right]^{1 / 2}} d v-14 \alpha \int_{0}^{1} \frac{f_{\varepsilon}(\alpha)-f_{\varepsilon}(\alpha v) v}{\left[F_{\varepsilon}(\alpha)-F_{\varepsilon}(\alpha v)\right]^{3 / 2}} d v \\
& -4 \alpha^{2} \int_{0}^{1} \frac{f_{\varepsilon}^{\prime}(\alpha)-f_{\varepsilon}^{\prime}(\alpha v) v^{2}}{\left[F_{\varepsilon}(\alpha)-F_{\varepsilon}(\alpha v)\right]^{3 / 2}} d v+6 \alpha^{2} \int_{0}^{1} \frac{\left[f_{\varepsilon}(\alpha)-f_{\varepsilon}(\alpha v) v\right]^{2}}{\left[F_{\varepsilon}(\alpha)-F_{\varepsilon}(\alpha v)\right]^{5 / 2}} d v,
\end{aligned}
$$

and compute that

$$
H_{\varepsilon}^{\prime}(\alpha)=\frac{1}{\alpha^{2}} \int_{0}^{\alpha} \frac{K_{\varepsilon}(\alpha, u)}{\left[\triangle F_{\varepsilon}\right]^{7 / 2}} d u
$$

where

$$
\begin{gathered}
K_{\varepsilon}(\alpha, u)=-20\left(\triangle F_{\varepsilon}\right)^{2}\left(\triangle f_{\varepsilon}\right)-22\left(\triangle F_{\varepsilon}\right)^{2}\left(\triangle \tilde{f}_{\varepsilon}\right)-4\left(\triangle F_{\varepsilon}\right)^{2}\left(\triangle \hat{f}_{\varepsilon}\right) \\
+33\left(\triangle F_{\varepsilon}\right)\left(\triangle f_{\varepsilon}\right)^{2}+18\left(\triangle F_{\varepsilon}\right)\left(\triangle f_{\varepsilon}\right)\left(\triangle \tilde{f}_{\varepsilon}\right)-15\left(\triangle f_{\varepsilon}\right)^{3}, \\
\triangle F_{\varepsilon}=F_{\varepsilon}(\alpha)-F_{\varepsilon}(u), \\
\triangle f_{\varepsilon}=\alpha f_{\varepsilon}(\alpha)-u f_{\varepsilon}(u), \\
\triangle \tilde{f}_{\varepsilon}=\alpha^{2} f_{\varepsilon}^{\prime}(\alpha)-u^{2} f_{\varepsilon}^{\prime}(u), \\
\triangle \hat{f}_{\varepsilon}=\alpha^{3} f_{\varepsilon}^{\prime \prime}(\alpha)-u^{3} f_{\varepsilon}^{\prime \prime}(u) .
\end{gathered}
$$


For $0<u<\alpha$, we let $\triangle_{1} \equiv \alpha-u, \triangle_{2} \equiv \alpha^{2}-u^{2}, \triangle_{3} \equiv \alpha^{3}-u^{3}, \triangle_{4} \equiv \alpha^{4}-u^{4}$, $A \equiv \varepsilon \triangle_{4}, B \equiv \sigma \triangle_{3}, C \equiv \tau \triangle_{2}, D \equiv \rho \triangle_{1}$. Then $\triangle_{1}, \triangle_{2}, \triangle_{3}, \triangle_{4}, A, B, D>0$ and $C \geq 0$. Since $f_{\varepsilon}(u)=-\varepsilon u^{3}+\sigma u^{2}+\tau u+\rho$ and by (3.5) (3.8), we obtain that

$$
\begin{gathered}
\triangle F_{\varepsilon}=-A / 4+B / 3+C / 2+D, \\
\triangle f_{\varepsilon}=-A+B+C+D, \\
\triangle \tilde{f}_{\varepsilon}=-3 A+2 B+C, \\
\triangle \hat{f}_{\varepsilon}=-6 A+2 B .
\end{gathered}
$$

To prove Lemma 3.3, we need the next technical Lemmas 3.4-3.6.

Lemma 3.4. If $0<\varepsilon<\left(\frac{\sigma^{3}}{27 \rho}\right)^{1 / 2}$, then for any $\alpha \in\left[\gamma_{\varepsilon}, \beta_{\varepsilon}\right)$ and $0<u<\alpha$, $3 A-B>0, B-3 D>0$ and $A-D>0$.

Proof of Lemma 3.4. For $0<\varepsilon<\left(\frac{\sigma^{3}}{27 \rho}\right)^{1 / 2}$, since $\sigma /(3 \varepsilon)=\gamma_{\varepsilon} \leq \alpha$ and $0<u<\alpha$, we have

$$
3 A>3 \varepsilon(\alpha-u) \alpha\left(\alpha^{2}+\alpha u+u^{2}\right) \geq 3 \varepsilon\left(\frac{\sigma}{3 \varepsilon}\right)(\alpha-u)\left(\alpha^{2}+\alpha u+u^{2}\right)=B
$$

and

$$
B>\sigma(\alpha-u)\left(\frac{\sigma}{3 \varepsilon}\right)^{2}=3(\alpha-u)\left(\frac{\sigma^{3}}{27 \varepsilon^{2}}\right)>3 D .
$$

Hence $3 A-B>0, B-3 D>0$ and $A-D>0$. The proof of Lemma 3.4 is complete.

Lemma 3.5. For any fixed positive $\varepsilon<\left(\frac{\sigma^{3}}{27 \rho}\right)^{1 / 2}, H_{\varepsilon}^{\prime}(\alpha)>0$ for all $\alpha \in\left[\gamma_{\varepsilon}, \beta_{\varepsilon}\right)$.

Proof of Lemma 3.5. For any fixed positive $\varepsilon<\left(\frac{\sigma^{3}}{27 \rho}\right)^{1 / 2}$, and for $\alpha \in\left[\gamma_{\varepsilon}, \beta_{\varepsilon}\right)$ and $0<u<\alpha$, since $A, B, D>0$ and $C \geq 0$, and by (3.4), (3.9) and Lemma 3.4 , we compute that

$$
\begin{aligned}
K_{\varepsilon}(\alpha, u)= & -20\left(\triangle F_{\varepsilon}\right)^{2}\left(\triangle f_{\varepsilon}\right)-22\left(\triangle F_{\varepsilon}\right)^{2}\left(\triangle \tilde{f}_{\varepsilon}\right)-4\left(\triangle F_{\varepsilon}\right)^{2}\left(\triangle \hat{f}_{\varepsilon}\right) \\
& +33\left(\triangle F_{\varepsilon}\right)\left(\triangle f_{\varepsilon}\right)^{2}+18\left(\triangle F_{\varepsilon}\right)\left(\triangle f_{\varepsilon}\right)\left(\triangle \tilde{f}_{\varepsilon}\right)-15\left(\triangle f_{\varepsilon}\right)^{3} \\
= & \frac{1}{72}\left(I_{1}+I_{2}+I_{3}+I_{4}+I_{5}\right),
\end{aligned}
$$

where

$$
\begin{gathered}
I_{1}=9 A^{3}+12 A^{2} B-2 A B^{2}>2 A B(3 A-B)>0, \\
I_{2}=504 A C D-468 C D^{2}+90 A C^{2}+168 B C D+72 C^{2} D \geq 468 C D(A-D) \geq 0, \\
I_{3}=138 B^{2} D+2646 A D^{2}-1248 B D^{2}-144 D^{3} \\
=138 B D(B-3 D)+144 D^{2}(A-D)+834 D^{2}(3 A-B)>0, \\
I_{4}=\left(207 A^{2}-168 A B+60 B^{2}\right) C \geq 0, \\
I_{5}=\left(1134 A^{2}-1356 A B+422 B^{2}\right) D>0 .
\end{gathered}
$$

So for any fixed positive $\varepsilon<\left(\frac{\sigma^{3}}{27 \rho}\right)^{1 / 2}$, and for $\alpha \in\left[\gamma_{\varepsilon}, \beta_{\varepsilon}\right)$ and $0<u<\alpha$, we have $K_{\varepsilon}(\alpha, u)>0$. Hence $H_{\varepsilon}^{\prime}(\alpha)=\frac{1}{\alpha^{2}} \int_{0}^{\alpha} \frac{K_{\varepsilon}(\alpha, u)}{\left[\triangle F_{\varepsilon}\right]^{7 / 2}} d u>0$ on $\left[\gamma_{\varepsilon}, \beta_{\varepsilon}\right)$ by (3.3). This completes the proof of Lemma 3.5. 
For any fixed $\alpha>0$, let

$$
I_{\alpha}=\left\{\varepsilon>0: \alpha \in\left(0, \beta_{\varepsilon}\right)\right\} .
$$

Since $\beta_{\varepsilon}$ is a continuous, strictly decreasing function of $\varepsilon>0$, and $\lim _{\varepsilon \rightarrow 0^{+}} \beta_{\varepsilon}=\infty$ and $\lim _{\varepsilon \rightarrow \infty} \beta_{\varepsilon}=0$, we obtain that $I_{\alpha}=(0, \varepsilon(\alpha))$, where $\alpha=\beta_{\varepsilon(\alpha)}$, and $\varepsilon(\alpha)$ is strictly decreasing in $\alpha$.

Lemma 3.6. For any fixed $\alpha>0, T_{\varepsilon}^{\prime}(\alpha)$ is a continuously differentiable, strictly increasing function of $\varepsilon \in I_{\alpha} \cup\{0\}$.

Proof of Lemma 3.6. First, for any fixed $\alpha>0$, it can be proved that $T_{\varepsilon}^{\prime}(\alpha)$ is a continuously differentiable function of $\varepsilon \in I_{\alpha} \cup\{0\}$. The proof is easy but tedious and we omit it.

Secondly, by (3.2), (3.5), (3.6), (3.9) and (3.10), we compute that

$$
\begin{aligned}
T_{\varepsilon}^{\prime}(\alpha) & =\frac{1}{\sqrt{2}} \int_{0}^{1} \frac{1}{\left[F_{\varepsilon}(\alpha)-F_{\varepsilon}(\alpha v)\right]^{1 / 2}} d v-\frac{\alpha}{2 \sqrt{2}} \int_{0}^{1} \frac{f_{\varepsilon}(\alpha)-f_{\varepsilon}(\alpha v) v}{\left[F_{\varepsilon}(\alpha)-F_{\varepsilon}(\alpha v)\right]^{3 / 2}} d v \\
& =\frac{1}{2 \sqrt{2} \alpha} \int_{0}^{\alpha} \frac{\varepsilon \frac{\triangle_{4}}{2}-\sigma \frac{\triangle_{3}}{3}+\rho \triangle_{1}}{\left[-\varepsilon \frac{\triangle_{4}}{4}+\sigma \frac{\triangle_{3}}{3}+\tau \frac{\triangle_{2}}{2}+\rho \triangle_{1}\right]^{3 / 2}} d u
\end{aligned}
$$

and

$$
\frac{\partial}{\partial \varepsilon} T_{\varepsilon}^{\prime}(\alpha)=\frac{1}{2 \sqrt{2} \alpha} \int_{0}^{\alpha} \frac{\triangle_{4}\left[3 \varepsilon \triangle_{4}+2 \sigma \triangle_{3}+12 \tau \triangle_{2}+42 \rho \triangle_{1}\right]}{48\left[\triangle F_{\varepsilon}\right]^{5 / 2}} d u>0 .
$$

So, for any fixed $\alpha>0, T_{\varepsilon}^{\prime}(\alpha)$ is a strictly increasing function of $\varepsilon \in I_{\alpha} \cup\{0\}$. This completes the proof of Lemma 3.6.

We are now in a position to prove Lemma 3.3.

Proof of Lemma 3.3. First, we prove that for any fixed $\varepsilon>0, T_{\varepsilon}(\alpha)$ is either a strictly increasing function or has a local maximum and a local minimum on $\left(0, \beta_{\varepsilon}\right)$.

For fixed $\varepsilon \geq\left(\frac{\sigma^{3}}{27 \rho}\right)^{1 / 2}$, we obtain that $T_{\varepsilon}(\alpha)$ is a strictly increasing function on $\left(0, \beta_{\varepsilon}\right)$ by Lemma 3.2(i). So we only need to consider the case $0<\varepsilon<\left(\frac{\sigma^{3}}{27 \rho}\right)^{1 / 2}$.

For any fixed positive $\varepsilon<\left(\frac{\sigma^{3}}{27 \rho}\right)^{1 / 2}$, by Lemma 3.1(ii) (resp. Lemma 3.5), we know that all (possible) critical points of $T_{\varepsilon}(\alpha)$ on $\left(0, \gamma_{\varepsilon}\right]$ (resp. on $\left[\gamma_{\varepsilon}, \beta_{\varepsilon}\right)$ ) are discrete. Moreover, since $\lim _{\alpha \rightarrow 0^{+}} T_{\varepsilon}(\alpha)=0$ and $\lim _{\alpha \rightarrow \beta_{\varepsilon}^{-}} T_{\varepsilon}(\alpha)=\infty$ from Lemma 3.1(i), we obtain that $T_{\varepsilon}^{\prime}(\alpha)$ changes sign an even number of times or infinitely many times. Assume that $T_{\varepsilon}(\alpha)$ is neither a strictly increasing function nor does it have exactly one local maximum and one local minimum on $\left(0, \beta_{\varepsilon}\right)$. Then there exist numbers $\alpha_{1}, \alpha_{2}, \alpha_{3} \in\left(0, \beta_{\varepsilon}\right)$ such that $\alpha_{1}<\alpha_{2}<\alpha_{3}$ are critical points of $T_{\varepsilon}(\alpha)$, $\alpha_{1}, \alpha_{3}$ are local maxima, and $\alpha_{2}$ is a local minimum. Thus $T_{\varepsilon}^{\prime \prime}\left(\alpha_{1}\right), T_{\varepsilon}^{\prime \prime}\left(\alpha_{3}\right) \leq 0$ and $T_{\varepsilon}^{\prime \prime}\left(\alpha_{2}\right) \geq 0$.

By Lemma 3.5, for any fixed positive $\varepsilon<\left(\frac{\sigma^{3}}{27 \rho}\right)^{1 / 2}$,

$$
H_{\varepsilon}(\alpha)=8 \sqrt{2} \alpha\left[T_{\varepsilon}^{\prime \prime}(\alpha)+\frac{3}{2 \alpha} T_{\varepsilon}^{\prime}(\alpha)\right]
$$

is a strictly increasing function on $\left[\gamma_{\varepsilon}, \beta_{\varepsilon}\right)$. Since $\alpha_{2} \geq \gamma_{\varepsilon}$ by Lemma 3.1(ii), we obtain that

$$
8 \sqrt{2} \alpha_{3} T_{\varepsilon}^{\prime \prime}\left(\alpha_{3}\right)=H_{\varepsilon}\left(\alpha_{3}\right)>H_{\varepsilon}\left(\alpha_{2}\right)=8 \sqrt{2} \alpha_{2} T_{\varepsilon}^{\prime \prime}\left(\alpha_{2}\right) \geq 0 .
$$


Therefore $T_{\varepsilon}^{\prime \prime}\left(\alpha_{3}\right)>0$. This contradicts that $T_{\varepsilon}^{\prime \prime}\left(\alpha_{3}\right) \leq 0$. So $T_{\varepsilon}(\alpha)$ is either a strictly increasing function or has exactly one local maximum and one local minimum on $\left(0, \beta_{\varepsilon}\right)$.

Next, suppose that $T_{\varepsilon}(\alpha)$ has exactly one local maximum $\alpha_{M}$ and one local minimum $\alpha_{m}$ for some fixed $\varepsilon>0$. Then $0<\alpha_{M}<\alpha_{m}<\beta_{\varepsilon}$ by Lemma 3.1(i). We next show that $T_{\varepsilon}(\alpha)$ has exactly two critical points $\alpha_{M}, \alpha_{m}$ on $\left(0, \beta_{\varepsilon}\right)$.

Assume that $\alpha_{*}$ is a critical point of $T_{\varepsilon}(\alpha)$ on $\left(0, \beta_{\varepsilon}\right)$, distinct from $\alpha_{M}, \alpha_{m}$. Then $T_{\varepsilon}^{\prime}\left(\alpha_{*}\right)=0$. If $\alpha_{*} \in\left(0, \alpha_{M}\right)$, there exist $0<\alpha_{1}<\alpha_{*}<\alpha_{2}<\alpha_{M}$ such that

$$
T_{\varepsilon}^{\prime}\left(\alpha_{1}\right), T_{\varepsilon}^{\prime}\left(\alpha_{2}\right)>0
$$

since all (possible) critical points of $T_{\varepsilon}(\alpha)$ on $\left(0, \beta_{\varepsilon}\right)$ are discrete. By Lemma 3.6, we obtain that $T_{\varepsilon}^{\prime}(\alpha)$ is a continuous, strictly increasing function of $\varepsilon \in I_{\alpha}$. Hence there exists a positive $\hat{\varepsilon}<\varepsilon$ such that

$$
T_{\hat{\varepsilon}}^{\prime}\left(\alpha_{1}\right)>0, T_{\hat{\varepsilon}}^{\prime}\left(\alpha_{*}\right)<0, T_{\hat{\varepsilon}}^{\prime}\left(\alpha_{2}\right)>0, T_{\hat{\varepsilon}}^{\prime}\left(\alpha_{M}\right)<0 .
$$

Thus $T_{\hat{\varepsilon}}(\alpha)$ has at least two local maxima on $\left(0, \beta_{\hat{\varepsilon}}\right)$, which contradicts the fact that $T_{\hat{\varepsilon}}(\alpha)$ has at most one local maximum on $\left(0, \beta_{\hat{\varepsilon}}\right)$. If $\alpha_{*} \in\left(\alpha_{m}, \beta_{\varepsilon}\right)$, the proof is similar, and consequently we omit it. If $\alpha_{*} \in\left(\alpha_{M}, \alpha_{m}\right)$, there exist $\alpha_{M}<\alpha_{3}<$ $\alpha_{*}<\alpha_{4}<\alpha_{m}$ such that

$$
T_{\varepsilon}^{\prime}\left(\alpha_{3}\right), T_{\varepsilon}^{\prime}\left(\alpha_{4}\right)<0
$$

since all (possible) critical points of $T_{\varepsilon}(\alpha)$ on $\left(0, \beta_{\varepsilon}\right)$ are discrete. By Lemma 3.6 again, there exists $\bar{\varepsilon}>\varepsilon$ such that $\bar{\varepsilon} \in I_{\alpha_{m}}$, and

$$
T_{\bar{\varepsilon}}^{\prime}\left(\alpha_{3}\right)<0, T_{\bar{\varepsilon}}^{\prime}\left(\alpha_{*}\right)>0, T_{\bar{\varepsilon}}^{\prime}\left(\alpha_{4}\right)<0, T_{\bar{\varepsilon}}^{\prime}\left(\alpha_{m}\right)>0 .
$$

Thus $T_{\bar{\varepsilon}}(\alpha)$ has at least two local minima on $\left(0, \beta_{\bar{\varepsilon}}\right)$, which contradicts the fact that $T_{\bar{\varepsilon}}(\alpha)$ has at most one local minimum on $\left(0, \beta_{\bar{\varepsilon}}\right)$.

So $T_{\varepsilon}(\alpha)$ has exactly two critical points $\alpha_{M}, \alpha_{m}$ on $\left(0, \beta_{\varepsilon}\right)$. This completes the proof of Lemma 3.3.

Let

$$
E=\left\{\begin{array}{l}
\varepsilon>0: T_{\varepsilon}(\alpha) \text { has exactly two critical points, } \\
\text { a local maximum and a local minimum, on }\left(0, \beta_{\varepsilon}\right)
\end{array}\right\} .
$$

We then prove, in the next lemma, that the set $E$ is open and connected, and hence $E$ is an open interval.

Lemma 3.7. The set $E$ is open and connected.

Proof of Lemma 3.7. We first show that $E$ is open. By Lemma 3.3, for any $\varepsilon>0$, $T_{\varepsilon}(\alpha)$ is either a strictly increasing function or has exactly two critical points, a local maximum and a local minimum, on $\left(0, \beta_{\varepsilon}\right)$. Thus

$$
\begin{aligned}
E & =\left\{\begin{array}{l}
\varepsilon>0: T_{\varepsilon}(\alpha) \text { has exactly two critical points, } \\
\text { a local maximum and a local minimum, on }\left(0, \beta_{\varepsilon}\right)
\end{array}\right\} \\
& =\left\{\varepsilon>0: T_{\varepsilon}^{\prime}(\alpha)<0 \text { for some } \alpha \in\left(0, \beta_{\varepsilon}\right)\right\} .
\end{aligned}
$$

If $\varepsilon_{0} \in E$, then $T_{\varepsilon_{0}}^{\prime}(\hat{\alpha})<0$ for some $\hat{\alpha} \in\left(0, \beta_{\varepsilon_{0}}\right)$. By Lemma 3.6, $T_{\varepsilon}^{\prime}(\hat{\alpha})$ is a continuous function of $\varepsilon \in I_{\hat{\alpha}}$. Thus $T_{\varepsilon}^{\prime}(\hat{\alpha})<0$ for $\varepsilon$ belonging to some open neighborhood of $\varepsilon_{0}$. So $E$ is open by (3.13).

We then show that $E$ is connected. Suppose that $E$ is not connected. Then there exist positive numbers $\varepsilon_{1}<\varepsilon_{2}<\varepsilon_{3}$ such that $\varepsilon_{1}, \varepsilon_{3} \in E$, but $\varepsilon_{2} \notin E$. Hence 
$T_{\varepsilon_{2}}^{\prime}(\alpha) \geq 0$ on $\left(0, \beta_{\varepsilon_{2}}\right)$ by (3.13). On the other hand, $T_{\varepsilon}^{\prime}(\alpha)$ is a strictly increasing function of $\varepsilon \in I_{\alpha}$ by Lemma 3.6. Thus

$$
T_{\varepsilon_{3}}^{\prime}(\alpha)>T_{\varepsilon_{2}}^{\prime}(\alpha) \geq 0 \text { for all } \alpha \in\left(0, \beta_{\varepsilon_{3}}\right) \subset\left(0, \beta_{\varepsilon_{2}}\right) .
$$

This contradicts that $\varepsilon_{3} \in E$. So $E$ is connected. This completes the proof of Lemma 3.7.

Lemma 3.8. $\left(0,\left(\frac{25}{32}\left(\frac{\sigma^{3}}{27 \rho}\right)\right)^{1 / 2}\right] \subset E$.

Proof of Lemma 3.8. First, we define an auxiliary function

$$
G_{\varepsilon}(u)=3 \int_{0}^{u} t f_{\varepsilon}(t) d t-u^{2} f_{\varepsilon}(u)=\frac{2}{5} \varepsilon u^{5}-\frac{1}{4} \sigma u^{4}+\frac{1}{2} \rho u^{2} .
$$

By applying [9, Lemmas 3.2 and 3.3], we obtain $T_{\varepsilon}^{\prime}(\alpha)<0$ if $G_{\varepsilon}(\alpha) \leq 0$ for some $\alpha \in\left(\gamma_{\varepsilon}, \beta_{\varepsilon}\right)$. By (3.13), this lemma holds if we can prove $G_{\varepsilon}(\alpha) \leq 0$ for some $\alpha \in\left(\gamma_{\varepsilon}, \beta_{\varepsilon}\right)$ for all $0<\varepsilon \leq\left(\frac{25}{32}\left(\frac{\sigma^{3}}{27 \rho}\right)\right)^{1 / 2}$.

So for $0<\varepsilon \leq\left(\frac{25}{32}\left(\frac{\sigma^{3}}{27 \rho}\right)\right)^{1 / 2}$, by (3.14), we obtain that $G_{\varepsilon}(0)=G_{\varepsilon}^{\prime}(0)=0$, $G_{\varepsilon}^{\prime}\left(\beta_{\varepsilon}\right)=-\beta_{\varepsilon}^{2} f_{\varepsilon}^{\prime}\left(\beta_{\varepsilon}\right)>0$, and

$$
G_{\varepsilon}^{\prime}(u) \begin{cases}>0 & \text { on }\left(0, p_{1}\right) \cup\left(p_{2}, \infty\right) \\ <0 & \text { on }\left(p_{1}, p_{2}\right)\end{cases}
$$

where $p_{1}$ and $p_{2}$ are two positive zeros of $G_{\varepsilon}^{\prime}(u)$ satisfying $0<p_{1}<\gamma_{\varepsilon}<p_{2}<\beta_{\varepsilon}$. So

$$
G_{\varepsilon}(u) \geq G_{\varepsilon}\left(p_{2}\right) \text { on }\left(\gamma_{\varepsilon}, \beta_{\varepsilon}\right)\left(=\left(\frac{\sigma}{3 \varepsilon}, \beta_{\varepsilon}\right)\right) .
$$

Let $g_{\varepsilon}(s) \equiv G_{\varepsilon}\left(s \gamma_{\varepsilon}\right)=G_{\varepsilon}\left(s\left(\frac{\sigma}{3 \varepsilon}\right)\right)$ for all $s \in[1, \infty)$. Then by (3.14), we compute that

$$
g_{\varepsilon}(s)=G_{\varepsilon}\left(s\left(\frac{\sigma}{3 \varepsilon}\right)\right)=\frac{s^{2} \sigma^{2}}{18 \varepsilon^{2}}\left[\frac{4 s^{3} \sigma^{3}}{135 \varepsilon^{2}}-\frac{s^{2} \sigma^{3}}{18 \varepsilon^{2}}+\rho\right]=\frac{s^{2} \sigma^{2}}{18 \varepsilon^{2}}\left[\rho+\frac{\sigma^{3}}{27 \varepsilon^{2}} g(s)\right],
$$

where $g(s)=\frac{4}{5} s^{3}-\frac{3}{2} s^{2}$. It is easy to see that $g(s)$ attains its minimum value $-\frac{25}{32}$ at $s=\frac{5}{4}$, and (3.16) implies that

$$
G_{\varepsilon}\left(p_{2}\right) \leq G_{\varepsilon}\left(\frac{5}{4}\left(\frac{\sigma}{3 \varepsilon}\right)\right)=g_{\varepsilon}\left(\frac{5}{4}\right)=\frac{25 \sigma^{2}}{288 \varepsilon^{2}}\left[\rho-\frac{25}{32}\left(\frac{\sigma^{3}}{27 \varepsilon^{2}}\right)\right] \leq 0
$$

since $0<\varepsilon \leq\left(\frac{25}{32}\left(\frac{\sigma^{3}}{27 \rho}\right)\right)^{1 / 2}$. So by [9] Lemmas 3.2 and 3.3], $T_{\varepsilon}^{\prime}\left(p_{2}\right)<0$ since $G_{\varepsilon}\left(p_{2}\right) \leq 0$ for all $\varepsilon \in\left(0,\left(\frac{25}{32}\left(\frac{\sigma^{3}}{27 \rho}\right)\right)^{1 / 2}\right]$. So $\left(0,\left(\frac{25}{32}\left(\frac{\sigma^{3}}{27 \rho}\right)\right)^{1 / 2}\right] \subset E$ by (3.13). This completes the proof of Lemma 3.8.

The following Lemma 3.9(i) determines the shape of $T_{\varepsilon=0}(\alpha)$ on $(0, \infty)$, and Lemma 3.9(ii) is a basic comparison theorem for the time-map formula. Lemma 3.9(i) follows from [13, Theorem 3.2] and Lemma 3.9(ii) by modifying [13, Theorems 2.3 and 2.4]. We omit the proofs.

Lemma 3.9. Consider (1.1), (1.2).

(i) $T_{\varepsilon=0}(\alpha)$ has exactly one critical point at some $\alpha_{0}$, a maximum, on $(0, \infty)$. Moreover, $\lim _{\alpha \rightarrow 0^{+}} T_{\varepsilon=0}(\alpha)=\lim _{\alpha \rightarrow \infty} T_{\varepsilon=0}(\alpha)=0$.

(ii) For any fixed $\alpha>0, T_{\varepsilon}(\alpha)$ is a continuous, strictly increasing function of $\varepsilon \in I_{\alpha} \cup\{0\}$. 


\section{Proofs of the MAIN REsults}

We first recall that a positive solution $u_{\varepsilon, \lambda}$ of (1.1), (1.2) is degenerate if $T_{\varepsilon}^{\prime}\left(\left\|u_{\varepsilon, \lambda}\right\|_{\infty}\right)=0$ and is nondegenerate if $T_{\varepsilon}^{\prime}\left(\left\|u_{\varepsilon, \lambda}\right\|_{\infty}\right) \neq 0$. Also, a degenerate positive solution $u_{\varepsilon, \lambda}$ of (1.1), (1.2) is of cusp type if $T_{\varepsilon}^{\prime \prime}\left(\left\|u_{\varepsilon, \lambda}\right\|_{\infty}\right)=0$ and $T_{\varepsilon}^{\prime \prime \prime}\left(\left\|u_{\varepsilon, \lambda}\right\|_{\infty}\right) \neq 0$; see [18, p. 214] and [17, p. 497].

We then prove our main results by applying the lemmas in Section 3.

Proof of Theorem 2.1. By (3.1) and Lemma 3.1(i), to prove Theorem 2.1, it suffices to prove that there exists a positive number $\tilde{\varepsilon}=\tilde{\varepsilon}(\sigma, \rho, \tau)$ such that the following parts (I)-(III) hold:

(I) For $0<\varepsilon<\tilde{\varepsilon}$, on $\left(0, \beta_{\varepsilon}\right), T_{\varepsilon}(\alpha)$ has exactly two critical points, a local maximum at some $\alpha_{\varepsilon}^{-}$and a local minimum at some $\alpha_{\varepsilon}^{+}\left(>\alpha_{\varepsilon}^{-}\right)$, satisfying $\lambda^{*}=\left(T_{\varepsilon}\left(\alpha_{\varepsilon}^{-}\right)\right)^{2}$ and $\lambda_{*}=\left(T_{\varepsilon}\left(\alpha_{\varepsilon}^{+}\right)\right)^{2}$.

(II) For $\varepsilon=\tilde{\varepsilon}, T_{\tilde{\varepsilon}}(\alpha)$ is a strictly increasing function and has exactly one critical point, at some $\tilde{\alpha}$, on $\left(0, \beta_{\tilde{\varepsilon}}\right)$. Moreover, $T_{\tilde{\varepsilon}}^{\prime}(\tilde{\alpha})=0, T_{\tilde{\varepsilon}}^{\prime}(\alpha)>0$ for $\alpha \in$ $\left(0, \beta_{\tilde{\varepsilon}}\right) \backslash\{\tilde{\alpha}\}, T_{\tilde{\varepsilon}}^{\prime \prime}(\tilde{\alpha})=0$ and $T_{\tilde{\varepsilon}}^{\prime \prime \prime}(\tilde{\alpha}) \neq 0$. (So (1.1), (1.2) has exactly one (cusp type) degenerate positive solution $u_{\tilde{\lambda}}$ with $\tilde{\lambda} \equiv\left(T_{\tilde{\varepsilon}}(\tilde{\alpha})\right)^{2}$ and $\tilde{\alpha}=\left\|u_{\tilde{\lambda}}\right\|_{\infty}$.)

(III) For $\varepsilon>\tilde{\varepsilon}, T_{\varepsilon}(\alpha)$ is a strictly increasing function and has no critical point on $\left(0, \beta_{\varepsilon}\right)$. Moreover, $T_{\varepsilon}^{\prime}(\alpha)>0$ for $\alpha \in\left(0, \beta_{\varepsilon}\right)$.

Note that the above parts (I)-(III) immediately imply the exact multiplicity result of positive solutions of (1.1), (1.2) for $0<\varepsilon<\tilde{\varepsilon}$ and the uniqueness result of positive solutions of (1.1), (1.2) for $\varepsilon \geq \tilde{\varepsilon}$. Moreover, ordering properties and asymptotic behaviors of positive solutions of (1.1), (1.2) in parts (I)-(III) can be obtained easily. We then prove parts (I)-(III) as follows.

By Lemma 3.2, we obtain that the set $E$ is nonempty and bounded above by $\left(\frac{\sigma^{3}}{27 \rho}\right)^{1 / 2}$. By Lemmas 3.7 and 3.8, $E=(0, \tilde{\varepsilon})$, where $\tilde{\varepsilon}=\sup E$ satisfies $\left(\frac{25}{32}\left(\frac{\sigma^{3}}{27 \rho}\right)\right)^{1 / 2}<\tilde{\varepsilon}<\left(\frac{\sigma^{3}}{27 \rho}\right)^{1 / 2}$. So, for $0<\varepsilon<\tilde{\varepsilon}$, on $\left(0, \beta_{\varepsilon}\right), T_{\varepsilon}(\alpha)$ has exactly two critical points, a local maximum at some $\alpha_{\varepsilon}^{-}$and a local minimum at some $\alpha_{\varepsilon}^{+}$ $\left(>\alpha_{\varepsilon}^{-}\right)$, satisfying $\lambda^{*}=\left(T_{\varepsilon}\left(\alpha_{\varepsilon}^{-}\right)\right)^{2}$ and $\lambda_{*}=\left(T_{\varepsilon}\left(\alpha_{\varepsilon}^{+}\right)\right)^{2}$. So part (I) holds.

For $\varepsilon>\tilde{\varepsilon}$, by Lemma 3.6 and (3.13), we obtain that

$$
T_{\varepsilon}^{\prime}(\alpha)>T_{\tilde{\varepsilon}}^{\prime}(\alpha) \geq 0 \text { for } \alpha \in\left(0, \beta_{\varepsilon}\right) \subset\left(0, \beta_{\tilde{\varepsilon}}\right),
$$

and hence $T_{\varepsilon}(\alpha)$ has no critical point on $\left(0, \beta_{\varepsilon}\right)$. So part (III) holds.

We prove the remaining part (II). For $\varepsilon=\tilde{\varepsilon}$, we know that

$$
T_{\tilde{\varepsilon}}^{\prime}(\alpha) \geq 0 \text { on }\left(0, \beta_{\tilde{\varepsilon}}\right) .
$$

We first prove the existence of a critical point of $T_{\tilde{\varepsilon}}(\alpha)$ on $\left(0, \beta_{\tilde{\varepsilon}}\right)$. Choose a sequence $\left\{\varepsilon_{n}\right\} \subset E=(0, \tilde{\varepsilon})$ such that $\varepsilon_{n} \nearrow \tilde{\varepsilon}$ as $n \rightarrow \infty$. Let $\alpha_{\varepsilon_{n}}^{-}<\alpha_{\varepsilon_{n}}^{+}$be two critical points of $T_{\varepsilon_{n}}(\alpha)$ on $\left(0, \beta_{\varepsilon_{n}}\right)$ for each $n \in \mathbb{N}$ (see Figure 7 ). Then by Lemma 3.6 again, we obtain that

$$
T_{\varepsilon_{n}}^{\prime}\left(\alpha_{\varepsilon_{n+1}}^{-}\right)<T_{\varepsilon_{n+1}}^{\prime}\left(\alpha_{\varepsilon_{n+1}}^{-}\right)=0 \text { and } T_{\varepsilon_{n}}^{\prime}\left(\alpha_{\varepsilon_{n+1}}^{+}\right)<T_{\varepsilon_{n+1}}^{\prime}\left(\alpha_{\varepsilon_{n+1}}^{+}\right)=0 .
$$

Hence $\alpha_{\varepsilon_{n}}^{-}<\alpha_{\varepsilon_{n+1}}^{-}<\alpha_{\varepsilon_{n+1}}^{+}<\alpha_{\varepsilon_{n}}^{+}$and

$$
\alpha_{\varepsilon_{n}}^{-}<\tilde{\alpha}^{-} \equiv \lim _{n \rightarrow \infty} \alpha_{\varepsilon_{n}}^{-} \leq \tilde{\alpha}^{+} \equiv \lim _{n \rightarrow \infty} \alpha_{\varepsilon_{n}}^{+}<\alpha_{\varepsilon_{n}}^{+} \text {for all } n \in \mathbb{N} .
$$

These imply that

$$
T_{\varepsilon_{n}}^{\prime}\left(\tilde{\alpha}^{-}\right), T_{\varepsilon_{n}}^{\prime}\left(\tilde{\alpha}^{+}\right)<0 \text { for all } n \in \mathbb{N} \text {. }
$$




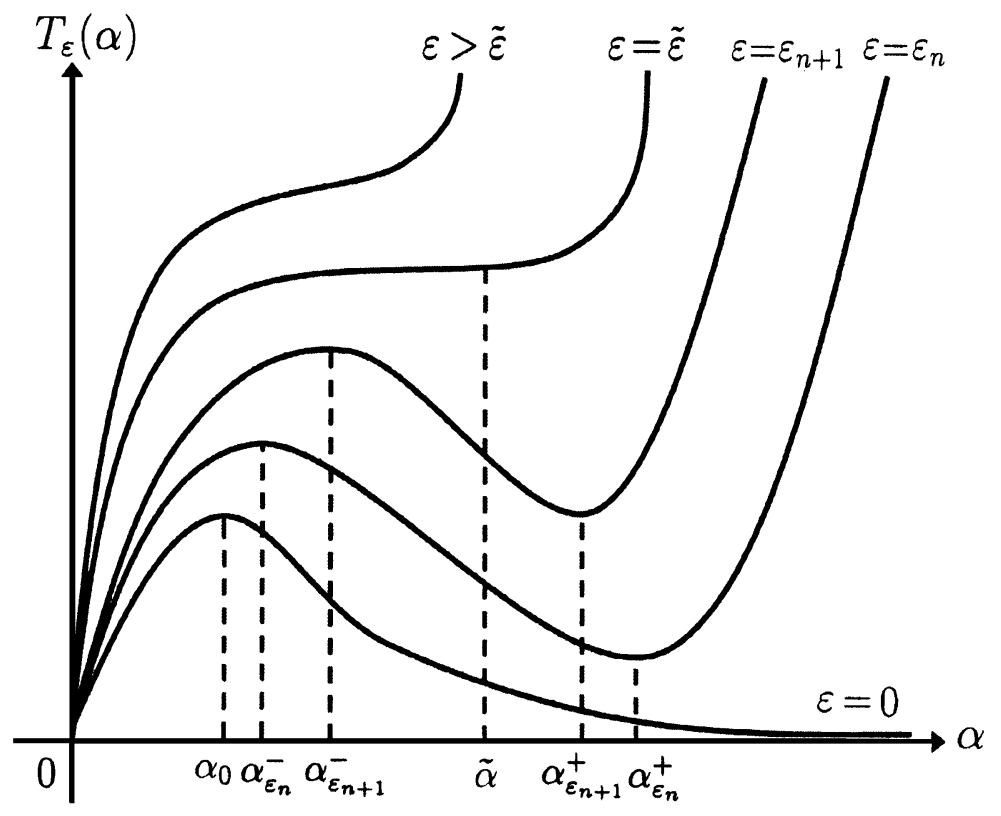

FIGURE 7 . Graphs of $T_{\varepsilon}(\alpha)$ for $\alpha \in\left(0, \beta_{\varepsilon}\right)$ with varying $\varepsilon \geq 0$.

By Lemma 3.6, we obtain that $T_{\varepsilon}^{\prime}(\alpha)$ is a continuous function of $\varepsilon \in I_{\alpha}$. Thus

$$
T_{\tilde{\varepsilon}}^{\prime}\left(\tilde{\alpha}^{-}\right)=\lim _{n \rightarrow \infty} T_{\varepsilon_{n}}^{\prime}\left(\tilde{\alpha}^{-}\right) \leq 0 \text { and } T_{\tilde{\varepsilon}}^{\prime}\left(\tilde{\alpha}^{+}\right)=\lim _{n \rightarrow \infty} T_{\varepsilon_{n}}^{\prime}\left(\tilde{\alpha}^{+}\right) \leq 0 .
$$

So $T_{\tilde{\varepsilon}}^{\prime}\left(\tilde{\alpha}^{-}\right)=T_{\tilde{\varepsilon}}^{\prime}\left(\tilde{\alpha}^{+}\right)=0$ by (4.1) and (4.2), and hence $T_{\tilde{\varepsilon}}(\alpha)$ has critical points at $\tilde{\alpha}^{-}, \tilde{\alpha}^{+}$on $\left(0, \beta_{\tilde{\varepsilon}}\right)$.

We then prove the uniqueness of the critical point of $T_{\tilde{\varepsilon}}(\alpha)$ on $\left(0, \beta_{\tilde{\varepsilon}}\right)$. That is, we prove that $\tilde{\alpha} \equiv \tilde{\alpha}^{-}=\tilde{\alpha}^{+}$is the unique critical point of $T_{\tilde{\varepsilon}}(\alpha)$ on $\left(0, \beta_{\tilde{\varepsilon}}\right)$. Suppose that $\hat{\alpha}<\bar{\alpha}$ are two critical points of $T_{\tilde{\varepsilon}}(\alpha)$ on $\left(0, \beta_{\tilde{\varepsilon}}\right)$. We know that all (possible) critical points of $T_{\varepsilon}(\alpha)$ on $\left(0, \beta_{\varepsilon}\right)$ are discrete as in the proof of Lemma 3.3. Hence there exist positive numbers $\alpha_{1}<\hat{\alpha}<\alpha_{2}<\bar{\alpha}$ such that

$$
T_{\tilde{\varepsilon}}^{\prime}\left(\alpha_{1}\right), T_{\tilde{\varepsilon}}^{\prime}\left(\alpha_{2}\right)>0 .
$$

By Lemma 3.6, we obtain that $T_{\varepsilon}^{\prime}(\alpha)$ is a continuous, strictly increasing function of $\varepsilon \in I_{\alpha}$. Hence there exists a positive $\hat{\varepsilon}<\tilde{\varepsilon}$ such that

$$
T_{\hat{\varepsilon}}^{\prime}\left(\alpha_{1}\right)>0, T_{\hat{\varepsilon}}^{\prime}(\hat{\alpha})<0, T_{\hat{\varepsilon}}^{\prime}\left(\alpha_{2}\right)>0, T_{\hat{\varepsilon}}^{\prime}(\bar{\alpha})<0 .
$$

Thus $T_{\hat{\varepsilon}}(\alpha)$ has at least two local maxima on $\left(0, \beta_{\hat{\varepsilon}}\right)$, which contradicts the fact that $\hat{\varepsilon} \in E$ and $T_{\hat{\varepsilon}}(\alpha)$ has exactly one local maximum on $\left(0, \beta_{\hat{\varepsilon}}\right)$. So $T_{\tilde{\varepsilon}}(\alpha)$ has at most one critical point on $\left(0, \beta_{\tilde{\varepsilon}}\right)$. By the above analysis,

$$
T_{\tilde{\varepsilon}}^{\prime}(\tilde{\alpha})=0 \text { and } T_{\tilde{\varepsilon}}^{\prime}(\alpha)>0 \text { for } \alpha \in\left(0, \beta_{\tilde{\varepsilon}}\right) \backslash\{\tilde{\alpha}\} .
$$

Next, if $T_{\tilde{\varepsilon}}^{\prime \prime}(\tilde{\alpha})>0\left(\operatorname{resp} . T_{\tilde{\varepsilon}}^{\prime \prime}(\tilde{\alpha})<0\right)$, then $T_{\tilde{\varepsilon}}(\alpha)$ has a local minimum (resp. a local maximum) at $\tilde{\alpha}$, which contradicts (4.3). So $T_{\tilde{\varepsilon}}^{\prime \prime}(\tilde{\alpha})=0$. By Lemma 3.1(ii), we have

$$
\alpha_{\varepsilon_{n}}^{+} \geq \gamma_{\varepsilon_{n}}>\gamma_{\tilde{\varepsilon}} \text { for all } n \in \mathbb{N} \text {, }
$$


and hence $\tilde{\alpha}=\lim _{n \rightarrow \infty} \alpha_{\varepsilon_{n}}^{+} \geq \gamma_{\tilde{\varepsilon}}$. By Lemma 3.5, $H_{\tilde{\varepsilon}}^{\prime}(\alpha)>0$ for all $\alpha \in\left[\gamma_{\tilde{\varepsilon}}, \beta_{\tilde{\varepsilon}}\right)$. So

$$
H_{\tilde{\varepsilon}}^{\prime}(\tilde{\alpha})=20 \sqrt{2} T_{\tilde{\varepsilon}}^{\prime \prime}(\tilde{\alpha})+8 \sqrt{2} \tilde{\alpha} T_{\tilde{\varepsilon}}^{\prime \prime \prime}(\tilde{\alpha})>0 .
$$

Therefore $T_{\tilde{\varepsilon}}^{\prime \prime \prime}(\tilde{\alpha})>0$ since $T_{\tilde{\varepsilon}}^{\prime \prime}(\tilde{\alpha})=0$. This completes the proof of part (II).

The proof of Theorem 2.1 is complete.

Proof of Theorem 2.3. Recall (3.1) with $\varepsilon \geq 0$,

$$
\sqrt{\lambda}=\frac{1}{\sqrt{2}} \int_{0}^{\alpha}\left[F_{\varepsilon}(\alpha)-F_{\varepsilon}(u)\right]^{-1 / 2} d u \equiv T_{\varepsilon}(\alpha) \text { for } 0<\alpha<\beta_{\varepsilon},
$$

where $\beta_{\varepsilon}$ is the unique positive zero of the cubic polynomial $f_{\varepsilon}(u)$ for $\varepsilon>0$ and $\beta_{\varepsilon=0}=\infty$. Thus, studying the exact number of positive solutions of (1.1), (1.2) for fixed $\lambda>0$ is equivalent to studying the number of roots of the equation $T_{\varepsilon}(\alpha)=\sqrt{\lambda}$ on $\left(0, \beta_{\varepsilon}\right)$ for varying $\varepsilon>0$. Since we have studied the behaviors of $T_{\varepsilon}(\alpha)$ for all varying $\varepsilon \geq 0$ (see the proofs of Theorem 2.1 and Lemma 3.9(i) and Figure 7$)$, there exist two positive numbers $\lambda_{0}\left(=\lambda_{0}(\sigma, \rho, \tau)\right)<\tilde{\lambda}(=\tilde{\lambda}(\sigma, \rho, \tau))$ such that the following parts (I)-(III) hold:

(I) For $0<\lambda \leq \lambda_{0}$, there exists a positive number $\varepsilon^{*}=\varepsilon^{*}(\lambda)$ such that the equation $T_{\varepsilon}(\alpha)=\sqrt{\lambda}$ has exactly three roots on $\left(0, \beta_{\varepsilon}\right)$ for $0<\varepsilon<\varepsilon^{*}$, exactly two roots on $\left(0, \beta_{\varepsilon}\right)$ for $\varepsilon=\varepsilon^{*}$, and exactly one root on $\left(0, \beta_{\varepsilon}\right)$ for $\varepsilon>\varepsilon^{*}$.

(II) For $\lambda_{0}<\lambda<\tilde{\lambda}$, there exist two positive numbers $\varepsilon_{*}\left(=\varepsilon_{*}(\lambda)\right)<\varepsilon^{*}$ $\left(=\varepsilon^{*}(\lambda)\right)$ such that the equation $T_{\varepsilon}(\alpha)=\sqrt{\lambda}$ has exactly three roots on $\left(0, \beta_{\varepsilon}\right)$ for $\varepsilon_{*}<\varepsilon<\varepsilon^{*}$, exactly two roots on $\left(0, \beta_{\varepsilon}\right)$ for $\varepsilon=\varepsilon_{*}$ and $\varepsilon=\varepsilon^{*}$, and exactly one root on $\left(0, \beta_{\varepsilon}\right)$ for $0<\varepsilon<\varepsilon_{*}$ and $\varepsilon>\varepsilon^{*}$.

(III) For $\lambda \geq \tilde{\lambda}$, the equation $T_{\varepsilon}(\alpha)=\sqrt{\lambda}$ has exactly one root on $\left(0, \beta_{\varepsilon}\right)$ for all $\varepsilon>0$.

Notice that $\lambda_{0}=\left(T_{\varepsilon=0}\left(\alpha_{0}\right)\right)^{2}$ and $\tilde{\lambda}=\left(T_{\tilde{\varepsilon}}(\tilde{\alpha})\right)^{2}$, where $\alpha_{0}$ is the unique critical point of $T_{\varepsilon=0}(\alpha)$ and $\tilde{\alpha}$ is the unique critical point of $T_{\tilde{\varepsilon}}(\alpha)$. Hence the above parts (I)-(III) immediately imply the exact multiplicity result of positive solutions of (1.1), (1.2) for $\lambda \in(0, \tilde{\lambda})$ and the uniqueness result of positive solutions of (1.1), (1.2) for $\lambda \geq \tilde{\lambda}$. Moreover, ordering properties and asymptotic behaviors of positive solutions of (1.1), (1.2) in parts (I)-(III) can be obtained easily.

The proof of Theorem 2.3 is complete.

Proof of Theorem 2.4. By Theorem 2.1, for any $\varepsilon \geq \tilde{\varepsilon}$, we obtain that (1.1), (1.2) has exactly one positive solution for all $\lambda>0$. In addition, for any $\varepsilon \in(0, \tilde{\varepsilon})$, there exist two positive numbers $\lambda_{*}(\varepsilon)<\lambda^{*}(\varepsilon)$ such that (1.1), (1.2) has exactly three positive solutions for $\lambda_{*}(\varepsilon)<\lambda<\lambda^{*}(\varepsilon)$, exactly two positive solutions for $\lambda=\lambda_{*}(\varepsilon)$ and $\lambda^{*}(\varepsilon)$, and exactly one positive solution for $0<\lambda<\lambda_{*}(\varepsilon)$ and $\lambda>\lambda^{*}(\varepsilon)$, where $\lambda_{*}(\varepsilon)=\left(T_{\varepsilon}\left(\alpha_{\varepsilon}^{+}\right)\right)^{2}$ and $\lambda^{*}(\varepsilon)=\left(T_{\varepsilon}\left(\alpha_{\varepsilon}^{-}\right)\right)^{2}$ in which $\alpha_{\varepsilon}^{-}<\alpha_{\varepsilon}^{+}$are two critical points of $T_{\varepsilon}(\alpha)$ on $\left(0, \beta_{\varepsilon}\right)$.

First, letting $\alpha_{\tilde{\varepsilon}}^{-}=\alpha_{\tilde{\varepsilon}}^{+} \equiv \tilde{\alpha}$, we prove that $\alpha_{\varepsilon}^{-}$(resp. $\alpha_{\varepsilon}^{+}$) is a continuous, strictly increasing (resp. strictly decreasing) function on $(0, \tilde{\varepsilon}]$ and $\lim _{\varepsilon \rightarrow 0^{+}} \alpha_{\varepsilon}^{-}=\alpha_{0}$ (resp. $\left.\lim _{\varepsilon \rightarrow 0^{+}} \alpha_{\varepsilon}^{+}=\infty\right)$ as follows. By similar arguments in the proof of Theorem 2.1, we obtain that $\alpha_{\varepsilon}^{-}$(resp. $\left.\alpha_{\varepsilon}^{+}\right)$is a strictly increasing (resp. strictly decreasing) function on $(0, \tilde{\varepsilon}]$. For any fixed $\alpha \in\left(\alpha_{0}, \tilde{\alpha}\right)$, by Theorem 2.1(ii) and Lemma 3.9(i), we obtain that

$$
T_{\varepsilon=0}^{\prime}(\alpha)<0 \text { and } T_{\tilde{\varepsilon}}^{\prime}(\alpha)>0 .
$$


Then by Lemma 3.6, $T_{\varepsilon}^{\prime}(\alpha)$ is a continuously differentiable, strictly increasing function of $\varepsilon \in[0, \tilde{\varepsilon}]$. This implies that there exists a unique $\varepsilon \in(0, \tilde{\varepsilon})$ such that $T_{\varepsilon}^{\prime}(\alpha)=0$. So

$$
\alpha_{\varepsilon}^{-}:(0, \tilde{\varepsilon}] \rightarrow\left(\alpha_{0}, \tilde{\alpha}\right] \text { is a strictly increasing, surjective function, }
$$

and hence $\alpha_{\varepsilon}^{-}$is a continuous function on $(0, \tilde{\varepsilon}]$ and $\lim _{\varepsilon \rightarrow 0^{+}} \alpha_{\varepsilon}^{-}=\alpha_{0}$. Similarly, we can prove that

$$
\alpha_{\varepsilon}^{+}:(0, \tilde{\varepsilon}] \rightarrow[\tilde{\alpha}, \infty) \text { is a strictly decreasing, surjective function, }
$$

and hence $\alpha_{\varepsilon}^{+}$is also a continuous function on $(0, \tilde{\varepsilon}]$ and $\lim _{\varepsilon \rightarrow 0^{+}} \alpha_{\varepsilon}^{+}=\infty$.

Secondly, let

$$
\lambda_{*}(0) \equiv 0, \lambda^{*}(0) \equiv \lambda_{0}=\left(T_{\varepsilon=0}\left(\alpha_{0}\right)\right)^{2} \text {, and } \lambda_{*}(\tilde{\varepsilon})=\lambda^{*}(\tilde{\varepsilon}) \equiv \tilde{\lambda}=\left(T_{\tilde{\varepsilon}}(\tilde{\alpha})\right)^{2} .
$$

By (4.4), (4.5), Lemma 3.6 and Lemma 3.9(ii), it can be proved that $\lambda^{*}=\left(T_{\varepsilon}\left(\alpha_{\varepsilon}^{-}\right)\right)^{2}$ and $\lambda_{*}=\left(T_{\varepsilon}\left(\alpha_{\varepsilon}^{+}\right)\right)^{2}$ satisfy

$$
\lambda^{*}(\varepsilon):[0, \tilde{\varepsilon}] \rightarrow\left[\lambda_{0}, \tilde{\lambda}\right] \text { is a continuous, strictly increasing function }
$$

and

$$
\lambda_{*}(\varepsilon):[0, \tilde{\varepsilon}] \rightarrow[0, \tilde{\lambda}] \text { is a continuous, strictly increasing function. }
$$

The proofs are easy but tedious, and hence we omit them. Moreover,

$$
\lim _{\varepsilon \rightarrow 0^{+}} \lambda^{*}(\varepsilon)=\lambda_{0}, \quad \lim _{\varepsilon \rightarrow 0^{+}} \lambda_{*}(\varepsilon)=0, \text { and } \lambda_{*}(\tilde{\varepsilon})=\lambda^{*}(\tilde{\varepsilon})=\tilde{\lambda} .
$$

The proof is easy but tedious, and hence we omit it.

Finally, by (4.6)-(4.8), $\lambda^{*}(\varepsilon)$ and $\lambda_{*}(\varepsilon)$ both have continuous inverse functions on $(0, \tilde{\varepsilon}]$. Indeed, by Theorem 2.3 and (3.1), $\varepsilon_{*}(\lambda)=\left(\lambda^{*}\right)^{-1}(\varepsilon)$ on $\left(\lambda_{0}, \tilde{\lambda}\right]$ and $\varepsilon^{*}(\lambda)=\left(\lambda_{*}\right)^{-1}(\varepsilon)$ on $(0, \tilde{\lambda}]$, where $\varepsilon_{*}(\tilde{\lambda})=\varepsilon^{*}(\tilde{\lambda}) \equiv \tilde{\varepsilon}$. So we obtain that

$$
\varepsilon^{*}(\lambda):(0, \tilde{\lambda}] \rightarrow(0, \tilde{\varepsilon}] \text { is a continuous, strictly increasing function }
$$

and

$$
\varepsilon_{*}(\lambda):\left(\lambda_{0}, \tilde{\lambda}\right] \rightarrow(0, \tilde{\varepsilon}] \text { is a continuous, strictly increasing function. }
$$

Moreover,

$$
\lim _{\lambda \rightarrow 0^{+}} \varepsilon^{*}(\lambda)=\lim _{\lambda \rightarrow \lambda_{0}^{+}} \varepsilon_{*}(\lambda)=0 .
$$

The proof of Theorem 2.4 is complete.

We prove Theorem 2.5 by applying Lemmas 3.3 and 3.8 .

Proof of Theorem 2.5. Consider (1.5), (1.6). Suppose that (1.7),

$$
\frac{16}{27}\left(\frac{a+b+c}{3}\right)^{3}<a b c<\left(\frac{a+b+c}{3}\right)^{3},
$$

holds. By Lemma 3.3, $T_{\varepsilon=1}(\alpha)$ is either a strictly increasing function or has exactly two critical points, a local maximum and a local minimum, on $\left(0, \beta_{\varepsilon=1}\right)$. Hence the bifurcation curve $S_{\varepsilon=1}$ is either monotone increasing or S-shaped on the $\left(\lambda,\|u\|_{\infty}\right)$ plane by (3.1). Thus, Theorem 2.5(ii) follows immediately. In the following, we then prove Theorem 2.5(i). To do this, suppose that (2.1),

$$
\frac{16}{27}\left(\frac{a+b+c}{3}\right)^{3}<a b c \leq \frac{25}{32}\left(\frac{a+b+c}{3}\right)^{3},
$$


holds. Notice that $G_{\varepsilon=1}(u)$ satisfy (3.14)-(3.17), and all analyses in the proof of Lemma 3.8 hold with $\sigma=a+b+c>0, \tau=a b+b c+c a=f^{\prime}(0)>0, \rho=a b c>0$. By (3.17),

$$
G_{\varepsilon=1}\left(p_{2}\right) \leq \frac{25 \sigma^{2}}{288}\left[\rho-\frac{25}{32}\left(\frac{\sigma^{3}}{27}\right)\right]=\frac{25(a+b+c)^{2}}{288}\left[a b c-\frac{25}{32}\left(\frac{a+b+c}{3}\right)^{3}\right] \leq 0
$$

if $a b c \leq \frac{25}{32}\left(\frac{a+b+c}{3}\right)^{3}$. By [9, Lemmas 3.2 and 3.3], $T_{\varepsilon=1}^{\prime}\left(p_{2}\right)<0$ if $a b c \leq \frac{25}{32}\left(\frac{a+b+c}{3}\right)^{3}$. Thus, by (2.1) and (3.13), $T_{\varepsilon=1}(\alpha)$ has exactly two critical points, a local maximum and a local minimum, on $\left(0, \beta_{\varepsilon=1}\right)$. So the bifurcation curve $S_{\varepsilon=1}$ is S-shaped on the $\left(\lambda,\|u\|_{\infty}\right)$-plane. The remaining parts of the results in Theorem 2.5(i), including the exact multiplicity result of positive solutions, follow immediately by (3.1). This completes the proof of Theorem 2.5(i).

The proof of Theorem 2.5 is complete.

\section{Conclusions}

5.1. A remark on the time-map techniques developed in this paper. To apply time-map techniques to study the shape of the bifurcation curve and the exact number of positive solutions of the Dirichlet problem

$$
\left\{\begin{array}{l}
u^{\prime \prime}(x)+\lambda f(u)=0,-1<x<1, \\
u(-1)=u(1)=0
\end{array}\right.
$$

the key points are to count the exact number of critical points and to determine whether they are either local minima or local maxima or inflection points of the time-map function

$$
T(\alpha) \equiv \frac{1}{\sqrt{2}} \int_{0}^{\alpha}[F(\alpha)-F(u)]^{-1 / 2} d u \text { for } \alpha=\|u\|_{\infty} \in I_{f},
$$

where $F(u) \equiv \int_{0}^{u} f(t) d t$ and $I_{f}$ is the interval of definition of $T(\alpha)$. To these problems, some feasible ideas originate from Smoller and Wasserman [19, Section $2]$ in which they applied estimates of the form

$$
T^{\prime \prime}(\alpha)+k T^{\prime}(\alpha)>0, \text { or }<0
$$

for some (nonzero) functions $k=k(\alpha)$. In particular, for the cubic polynomial $f(u)=(u-a)(u-b)(c-u)$ satisfying $0 \leq a<b<c$ and $c+a>2 b$, to show that $T(\alpha)$ has exactly one critical point, a minimum, for $\alpha \in \tilde{I}_{f}$ for some interval $\tilde{I}_{f} \subset I_{f}$, Smoller and Wasserman chose the functions

$$
k=k(\alpha)=\frac{3}{\alpha} \text { and } k=k(\alpha)=\frac{2}{\alpha}
$$

for the case $a=0$ and $a>0$, respectively (see [19, p. 273 and p. 275]). Afterward, the estimate $T^{\prime \prime}(\alpha)+k T^{\prime}(\alpha)>0$ with $k(\alpha)$ chosen in (5.1), and the estimate $T^{\prime \prime}(\alpha)+k T^{\prime}(\alpha)<0$ with $k(\alpha)$ chosen in [19, (2.12)] were studied and extended to various forms for different problems by many researchers; see e.g. [4, 5, 7, 14, 21, 22, . However, these estimates are inapplicable in studying the global bifurcation and exact multiplicity of positive solutions for our problem (1.1), (1.2). Even for (1.3) with $\sigma=\tau=\rho=1$, these estimates are inapplicable in solving the long-standing conjecture for global bifurcation of bifurcation curves $S_{\varepsilon}$ for all $\varepsilon>0$ since the paper of Crandall and Rabinowitz [3] published in 1973. 
In this paper, we developed new time-map techniques to prove the global bifurcation results. In the proof of our key Lemma 3.3, the novelty used there is to define the auxiliary function

$$
H_{\varepsilon}(\alpha)=8 \sqrt{2} \alpha\left[T_{\varepsilon}^{\prime \prime}(\alpha)+\frac{3}{2 \alpha} T_{\varepsilon}^{\prime}(\alpha)\right]
$$

and then to study its variations. More precisely, we proved that for any fixed positive $\varepsilon<\left(\frac{\sigma^{3}}{27 \rho}\right)^{1 / 2}, H_{\varepsilon}(\alpha)$ is a strictly increasing function on $\left[\gamma_{\varepsilon}, \beta_{\varepsilon}\right)$. Thus we were able to prove that the terms of the sequence $\left\{T_{\varepsilon}^{\prime \prime}\left(\alpha_{n}\right)\right\}$ change sign at most once as the $\left\{\alpha_{n}\right\}$ increase, where the $\left\{\alpha_{n}\right\}$ are all (possible) critical points of $T_{\varepsilon}(\alpha)$. So for any fixed $\varepsilon>0, T_{\varepsilon}(\alpha)$ is either a strictly increasing function or has exactly two critical points, a local maximum and a local minimum, on $\left(0, \beta_{\varepsilon}\right)$. To the best of our knowledge, there is no paper in the literature where one can find a study of the variations of an expression such as our $H_{\varepsilon}(\alpha)$.

\subsection{Three open problems with observed similar global bifurcation results}

in Theorem 2.1. In this subsection we state three open problems in one space variable with observed similar global bifurcation results in Theorem 2.1; see Figures 2 and 3. Further investigations are needed to determine the exact shape of the bifurcation curves of these three open problems.

(i) The problem (1.5), (1.6) satisfying (2.2). In Theorem 2.3(ii) for (1.5), (1.6) satisfying (2.2), it was proved that the bifurcation curve $S_{\varepsilon=1}$ is either Sshaped or monotone increasing on the $\left(\lambda,\|u\|_{\infty}\right)$-plane, and the problem has at most three positive solutions for each $\lambda>0$. However, the exact shape of the bifurcation curve $S_{\varepsilon=1}$ still remains undetermined. We conjecture that, for fixed numbers $a<b<0$, there exists $\tilde{c}=\tilde{c}(a, b)>-a-b>0$ such that, on the $\left(\lambda,\|u\|_{\infty}\right)$-plane, the bifurcation curve $S_{\varepsilon=1}$ of (1.5), (1.6) is S-shaped for $-a-b<c<\tilde{c}$ and is monotone increasing for $c \geq \tilde{c}$.

(ii) The cubic autocatalytic problem

$$
\left\{\begin{array}{l}
u^{\prime \prime}(x)+\lambda\left[(u+k)^{2}-(u+k)^{3}\right] \\
=u^{\prime \prime}(x)+\lambda\left[k^{2}-k^{3}+\left(2 k-3 k^{2}\right) u+(1-3 k) u^{2}-u^{3}\right]=0, \quad-1<x<1, \\
u(-1)=u(1)=0, \quad \lambda>0, k \in(0,1) .
\end{array}\right.
$$

This problem arises from an autocatalytic chemical reaction. We refer to [8, 24] for more background of this problem and its generalizations. It is easy to see that, for $k \in(0,1)$, there exists a positive number $\beta_{k}<1$ which is the unique positive zero of the cubic polynomial $f_{k}(u) \equiv k^{2}-k^{3}+(2 k-$ $\left.3 k^{2}\right) u+(1-3 k) u^{2}-u^{3}$ such that $f_{k}$ satisfies $f_{k}(0)=k^{2}-k^{3}>0, f_{k}(u)>0$ on $\left(0, \beta_{k}\right)$ and $f_{k}\left(\beta_{k}\right)=0$. For $1 / 9 \leq k<1$, it is easy to show that the bifurcation curve of positive solutions of $(5.2)$ is monotone increasing; see [24, Section 5, Remark 1]. While $0<k<1 / 9$, by applying Theorem 2.1, the bifurcation curve of positive solutions of (5.2) is either S-shaped or monotone increasing on the $\left(\lambda,\|u\|_{\infty}\right)$-plane, and hence (5.2) has at most three positive solutions for each $\lambda>0$. Recently, Zhao, Wang and Shi 24. Section 5, Remark 1] conjectured that there exists $\tilde{k} \in(0,1 / 9)$ such that, on the $\left(\lambda,\|u\|_{\infty}\right)$-plane, the bifurcation curve of (5.2) is S-shaped for $0<k<\tilde{k}$ and is monotone increasing for $k \geq \tilde{k}$. 
(iii) The perturbed Gelfand problem

$$
\left\{\begin{array}{l}
u^{\prime \prime}(x)+\lambda \exp \left(\frac{u}{1+\varepsilon u}\right)=0, \quad-1<x<1, \\
u(-1)=u(1)=0, \quad \lambda, \varepsilon>0,
\end{array}\right.
$$

where $\lambda>0$ is the Frank-Kamenetskii parameter, $\varepsilon$ is the reciprocal activation energy, $u(x)$ is the dimensionless temperature, and the reaction term $\exp \left(\frac{u}{1+\varepsilon u}\right)$ is the temperature dependence obeying the Arrhenius reactionrate law. This is the one-dimensional case of a problem of some importance in the theory of combustion, which has been extensively investigated by many authors. We refer to [1, 6] for more background of this problem. It is easy to show that, on the $\left(\lambda,\|u\|_{\infty}\right)$-plane, the bifurcation curve of positive solutions of (5.3) is monotone increasing for $\varepsilon \geq 1 / 4=0.25$; see [2, p. 482]. Brown et al. [2, p. 482] showed that the bifurcation curve of positive solutions of (5.3) is S-like shaped for $0<\varepsilon \leq \hat{\varepsilon}=1 / 4.25 \approx 0.235$. Wang [21, Theorem 1] used time-map techniques to prove that the bifurcation curve of positive solutions of (5.3) is S-shaped for $0<\varepsilon \leq \bar{\varepsilon} \approx 1 / 4.4967 \approx 0.222$. This upper bound for $\varepsilon$ was improved to $1 / 4.35 \approx 0.230$ by Korman and Li [11, Theorem 3.1] by applying a bifurcation theorem of Crandall and Rabinowitz [3]. This upper bound for $\varepsilon$ was further improved recently to $1 / 4.166 \approx 0.240$ by Hung and Wang [9, Theorem 2.2].

For (5.3), it was a long-standing conjecture (2, 11, 12, 18, 21]) that there exists $\tilde{\varepsilon}>0$ such that, on the $\left(\lambda,\|u\|_{\infty}\right)$-plane, the bifurcation curve of positive solutions is S-shaped for $0<\varepsilon<\tilde{\varepsilon}$ and is monotone increasing for $\varepsilon \geq \tilde{\varepsilon}$. For this conjecture, Korman, Li and Ouyang [12] gave a computerassisted proof. Numerical calculations show that $\tilde{\varepsilon} \approx 1 / 4.07 \approx 0.248$.

\section{ACKNOWLEDGMENTS}

The authors thank Dr. Idris Addou and Professors Junping Shi and Hwai-Chiuan Wang for giving many illuminating comments and suggestions. The authors also thank the referee for a careful reading of the manuscript and valuable suggestions. Most of the computation in this paper has been checked using the symbolic manipulator Mathematica 6.0.

\section{REFERENCES}

[1] J. Bebernes, D. Eberly, Mathematical Problems from Combustion Theory, Springer-Verlag, New York, 1989. MR.1012946(91d:35165)

[2] K.J. Brown, M.M.A. Ibrahim, R. Shivaji, S-shaped bifurcation curves, Nonlinear Anal. 5 (1981), 475-486. MR0613056 (82h:35007)

[3] M.G. Crandall, P.H. Rabinowitz, Bifurcation, perturbation of simple eigenvalues and linearized stability, Arch. Rational Mech. Anal. 52 (1973), 161-180. MR0341212 (49:5962)

[4] J.I. Díaz, J. Hernández, Global bifurcation and continua of nonnegative solutions for a quasilinear elliptic problem, C. R. Acad. Sci. Paris Sér. I Math. 329 (1999), 587-592. MR1717114 (2000h:34028)

[5] J.I. Díaz, J. Hernández, F.J. Mancebo, Branches of positive and free boundary solutions for some singular quasilinear elliptic problems, J. Math. Anal. Appl. 352 (2009), 449-474. MR2499916 (2010h:35163)

[6] Y. Du, Exact multiplicity and $S$-shaped bifurcation curve for some semilinear elliptic problems from combustion theory, SIAM J. Math. Anal. 32(2000), 707-733. MR 1814735 (2001m:35105) 
[7] M. Feng, X. Zhang, W. Ge, Exact number of solutions for a class of two-point boundary value problems with one-dimensional p-Laplacian, J. Math. Anal. Appl. 338 (2008), 784-792. MR.2386460 (2009d:34039)

[8] P. Gray, S.K. Scott, Chemical Oscillations and Instabilities: Nonlinear Chemical Kinetics, Clarendon Press, Oxford, 1990. MR.1098081 (92b:80016)

[9] K.-C. Hung, S.-H. Wang, A theorem on S-shaped bifurcation curve for a positone problem with convex-concave nonlinearity and its applications to the perturbed Gelfand problem, $J$. Differential Equations 251 (2011), 223-237. MR2800152

[10] J.P. Kernevez, D. Thomas, Numerical analysis and control of some biochemical systems, Appl. Math. Optim. 1 (1975), 222-285. MR0403716 (53:7527)

[11] P. Korman, Y. Li, On the exactness of an S-shaped bifurcation curve, Proc. Amer. Math. Soc. 127 (1999), 1011-1020. MR.1610804 (99f:34027)

[12] P. Korman, Y. Li, T. Ouyang, Computing the location and the direction of bifurcation, Mathematical Research Letters 12 (2005), 933-944. MR2189251(2006h:34079)

[13] T. Laetsch, The number of solutions of a nonlinear two point boundary value problem, Indiana Univ. Math. J. 20 (1970), 1-13. MR0269922 (42:4815)

[14] R. Logan, Positive solutions to a system of differential equations modeling a competitive interactive system with nonlogistic growth rates, Differential Integral Equations 10 (1997), 929-945. MR 1741759 (2000m:34100)

[15] C. Lu, Multiple steady states in a biochemical system, SIAM J. Math. Anal. 18 (1987), 1771-1783. MR.0911662 (88k:92032)

[16] C. Lu, Global bifurcation of steady-state solutions on a biochemical system, SIAM J. Math. Anal. 21 (1990), 76-84. MR1032728 (91a:35025)

[17] J. Shi, Persistence and bifurcation of degenerate solutions, J. Funct. Anal. 169 (1999), 494531. MR:1730558 (2001h:47115)

[18] J. Shi, Multi-parameter bifurcation and applications, in: H. Brezis, K.C. Chang, S.J. Li, P. Rabinowitz (Eds.), ICM 2002 Satellite Conference on Nonlinear Functional Analysis: Topological Methods, Variational Methods and Their Applications, World Scientific, Singapore, 2003, pp. 211-222. MR2011699 (2004j:35026)

[19] J. Smoller, A. Wasserman, Global bifurcation of steady-state solutions, J. Differential Equations 39 (1981), 269-290. MR0607786 (82d:58056)

[20] K. Tanaka, S. Murashige, S. Oishi, On necessary and sufficient conditions for numerical verification of double turning points, Numer. Math. 97 (2004), 537-554. MR2059468 (2005m:65309)

[21] S.-H. Wang, On S-shaped bifurcation curves, Nonlinear Anal. 22 (1994), 1475-1485. MR.1285087(96d:34021)

[22] S.-H. Wang, D.-M. Long, An exact multiplicity theorem involving concave-convex nonlinearities and its application to stationary solutions of a singular diffusion problem, Nonlinear Anal. 44 (2001), 469-486. MR1822096 (2002b:34042)

[23] S.-H. Wang, T.-S. Yeh, A theorem on reversed S-shaped bifurcation curves for a class of boundary value problems and its application, Nonlinear Anal. 71 (2009), 126-140. MR2518020(2010e:34061)

[24] Y. Zhao, Y. Wang, J. Shi, Exact multiplicity of solutions and S-shaped bifurcation curve for a class of semilinear elliptic equations, J. Math. Anal. Appl. 331 (2007), 263-278. MR2306004 (2008e:35081)

Department of Mathematics, National Tsing Hua University, Hsinchu, Taiwan 300, Republic of China

E-mail address: kchung@mx.nthu.edu.tw

Department of Mathematics, National Tsing Hua University, Hsinchu, Taiwan 300, Republic of ChinA

E-mail address: shwang@math.nthu.edu.tw 